\title{
Signal-Level Information Fusion for Less Constrained Iris Recognition Using Sparse-Error Low Rank Matrix Factorization
}

\author{
Yang Hu, Konstantinos Sirlantzis, and Gareth Howells
}

\begin{abstract}
Iris recognition systems working in less constrained environments with the subject at-a-distance and on-the-move suffer from the noise and degradations in the iris captures. These noise and degradations significantly deteriorate iris recognition performance. In this paper, we propose a novel signal-level information fusion method to mitigate the influence of noise and degradations for less constrained iris recognition systems. The proposed method is based on low rank approximation (LRA). Given multiple noisy captures of the same eye, we assume that: 1) the potential noiseless images lie in a low rank subspace and 2) the noise is spatially sparse. Based on these assumptions, we seek an LRA of noisy captures to separate the noiseless images and noise for information fusion. Specifically, we propose a sparse-error low rank matrix factorization model to perform LRA, decomposing the noisy captures into a low rank component and a sparse error component. The low rank component estimates the potential noiseless images, while the error component models the noise. Then, the low rank and error components are utilized to perform signal-level fusion separately, producing two individually fused images. Finally, we combine the two fused images at the code level to produce one iris code as the final fusion result. Experiments on benchmark data sets demonstrate that the proposed signal-level fusion method is able to achieve a generally improved iris recognition performance in less constrained environment, in comparison with the existing iris recognition algorithms, especially for the iris captures with heavy noise and low quality.
\end{abstract}

Index Terms - Iris recognition, less constrained environment, information fusion.

\section{INTRODUCTION}

B IOMETRIC systems are widely used for automatic authentication of human using various physiological and behavioural characteristics, for example, fingerprint, face, iris, palm print, finger vein, gait, voice, retina, etc. Among these biometric traits, iris has shown a high reliability due to the stability and high degree of freedom of iris patterns. State-of-theart iris recognition algorithms [1]-[6] have reported promising

Manuscript received July 15, 2015; revised February 8, 2016; accepted March 3, 2016. Date of publication March 14, 2016; date of current version April 12, 2016. The associate editor coordinating the review of this manuscript and approving it for publication was Prof. Kar-Ann Toh.

The authors are with the School of Engineering and Digital Arts, University of Kent, Canterbury CT2 7NT, U.K. (e-mail: yh94@kent.ac.uk; k.sirlantzis@kent.ac.uk; w.g.j.howells@kent.ac.uk).

Color versions of one or more of the figures in this paper are available online at http://ieeexplore.ieee.org.

Digital Object Identifier 10.1109/TIFS.2016.2541612 performance with iris images captured in near-infrared (NIR) wavelength and under a controlled environment. Recently, significant research effort [7]-[24] has been devoted to improve the usability and practicality of iris recognition technology by allowing the iris images to be captured in less constrained environment, with the subject at-a-distance and on-the-move. In less constrained environment, the main challenge is the noise and degradations in iris captures. The captures usually suffer from a low resolution, together with some noise like motion blur, specular reflection, off-angle, eyelids and eyelashes occlusion, etc (in this paper, we simply use the term 'noise' to refer to all these noise and degradations, following many of the papers in the literature on less constrained iris recognition, like [7], [11], [12], and [14]-[16]). Therefore, a critical problem of iris recognition under less constrained environment is how to overcome the influence of noise in the iris captures.

In recent researches, information fusion has shown its power to handle the above problem [19]-[24]. The idea is to fuse the information in multiple captures to overcome the information loss and noise. In [19], Hollingsworth et al. extract images from iris videos and fuse them at the signal level by mean fusion. The results are compared to score level fusion. Based on the signal level fusion in [19], super-resolution methods [20], [21] are proposed for iris resolution enhancement. In [20] and [21], multiple iris captures are fused by weighted mean methods. The weights can be calculated using either individual focus estimation [20] or the combination of multiple quality measures [21]. Also, some researches [22]-[25] propose to fuse the information at the feature level, since features are more directly related to the iris recognition performance.

Although the aforementioned information fusion methods show their effectiveness for less constrained iris recognition, some problems still exist. One problem is that, most of the existing methods use original iris captures for fusion, and they do not distinguish between the clear iris pixels and noise pixels in the fusion process. It is possible to lead to an impaired performance. In less constrained environment, the iris captures are often corrupted by noise. The noise not only influences the original signal, but also embeds in the feature space [15]. In the fusion process, without distinguishing noise pixels from 


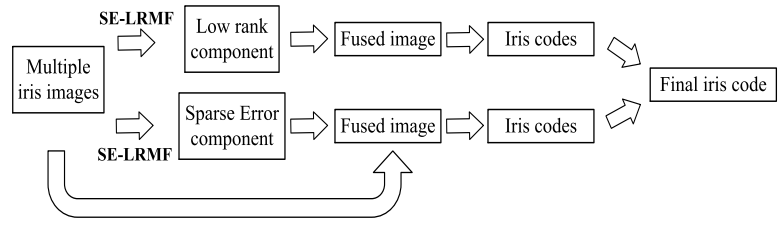

Fig. 1. An illustration of the proposed signal level fusion method.

clear iris pixels, the fusion result is possible to be influenced by the noise embedded in the signals and features. It degrades the performance. Therefore, we expect that an information fusion algorithm considering clear iris pixels and noise pixels separately is able to achieve a better performance.

With the above motivation, a key problem is how to seek clear iris pixels from multiple noisy iris captures. Recently, low rank approximation (LRA) [26]-[29] has shown its power to solve this problem. LRA algorithms assume that given multiple noisy observations, the noiseless signals lie in a low rank subspace. Based on this assumption, they seek the LRA of the original noisy observations as the estimation of noiseless signals. The above assumption of LRA is likely to hold for iris images of the same eye, due to the inherent stability of iris patterns. Thus, it is reasonable to utilize LRA to distinguish between clear iris pixels and noise pixels, designing an information fusion method that considers the clear iris pixels and noise pixels separately for iris recognition.

In this paper, we propose a novel signal-level information fusion method for less constrained iris recognition. The method is based on a sparse-error low rank matrix factorization (SE-LRMF) model which is proposed to seek a low rank approximation of iris images to distinguish between clear iris pixels and noise pixels. The flowchart of the proposed method is shown in Fig. 1. Firstly, given multiple iris captures of the same eye, we compute a low rank component and a sparse error component using SE-LRMF. The low rank component estimates the clear iris pixels, and the sparse error component is viewed as noise pixels. Then, the low rank component (clear iris pixels) and sparse error component (noise pixels) are considered individually for signal level fusion. The low rank component is fused straightforwardly, while the sparse error component is utilized to perform a weighted fusion of the original iris captures. Such a scheme leads to two fused images: one using low rank component, the other one using sparse error component and the original iris captures. Finally, we extract two iris codes from the two fused images, and the two iris codes are combined to produce a single iris code as the final fusion result.

Contributions: Our contributions are as follows. First, we propose a novel model named sparse-error low rank matrix factorization (SE-LRMF) for low rank approximation to separate clear iris pixels and noise pixels in multiple iris captures. SE-LRMF uses low rank matrix factorization to model the clear iris pixels, and it uses a sparse error term to estimate the noise pixels. We propose an alternating algorithm to solve SE-LRMF. Experimentally, we show that for iris images, SE-LRMF achieves a more stable and very competitive performance compared to existing low rank approximation algorithms.
Second, based on SE-LRMF, we propose a signal-level information fusion method considering the clear iris pixels and noise pixels separately. It consists of a low rank mean (LRM) fusion using clear iris pixels, and an error weighted mean (EWM) fusion using noise pixels and the original iris captures. We demonstrate that not only clear iris pixels can be straightforwardly fused via LRM, but also noise pixels are able to contribute to the signal level fusion by EWM.

Third, we combine the fusion results of LRM and EWM at the code level to produce a single iris code. In our experiments, we find that such code level combination leads to an improved performance in comparison to the individual fusion results of either LRM or EWM.

The remainder of the paper is organized as follows. In section II, we revisit the related works to information fusion in iris recognition, and low rank approximation. In section III, we present the proposed signal level fusion method. In section IV, we report the results of experimental analysis and comparisons. Finally in section V, we conclude the paper.

\section{RELATED WORK}

\section{A. Information Fusion in Iris Recognition}

Existing information fusion methods for iris recognition can be categorized into score level fusion, signal level fusion and feature level fusion. Given a set of gallery iris codes and a set of probe iris codes, score level fusion produces one matching score by fusing the matching scores between all the combinations of gallery and probe iris codes. Hollingsworth et al. [19] reports the performance of minimum fusion and mean fusion of matching scores on NIR iris videos.

Signal level fusion combines multiple iris captures to be one image, and iris codes are extracted from the fused image for matching. In [19], Hollingsworth et al. fuse iris images in video captures by mean and median fusion. As reported in [19], signal level fusion leads to better performance than no fusion, and mean fusion performs better than median fusion. The mean fusion method in [19] essentially assigns equal importance to all the pixels in all the iris captures. Different from this, weighted mean fusion methods are proposed in [20] and [21]. The weights are calculated based on image quality measures. The iris captures with higher quality are assigned higher weights. In [20], Nguyen $e t$ al. use focus score as quality measure. In [21], a better performance is achieved by combining multiple quality scores to produce a quality measure. The considered quality scores include focus, offangle, illumination variance and motion. Compared to score level fusion, signal level fusion has significant advantage in speed and memory cost. However, existing signal level fusion methods directly use the original iris captures for fusion. In less constrained environment, the fusion results are likely to be influenced by the noise in the original iris captures, although the noise level can be mitigated by fusion.

Feature level fusion aims to estimate the iris code of a clear and high-resolution iris image using the iris codes extracted from multiple noisy and low-resolution iris captures. The key step of feature level fusion is to learn the relationship between the iris codes of noiseless high-resolution iris images 
and noisy low-resolution iris images. Nguyen et al. [22] formulate feature level fusion in a Bayesian framework using PCA features (eigen-iris), and it can be solved by maximum a posteriori estimation approach. In [23] and [25], Nguyen et al. demonstrate that the similar Bayesian framework can be adapted to Gabor feature domain which is the most widely used feature in iris recognition. Liu et al. [24] propose to use a Markov network model for feature level fusion. This model considers the high resolution iris code as the hidden node of a Markov network, and it is computed using multiple low resolution iris codes served as the observations. Feature level fusion performs generally better than score level and signal level fusion, since the features (iris codes) are more directly related to the performance of iris recognition. However, most feature level fusion methods require a learning stage to build the relationship between high resolution and low resolution features, and such relationship can be data dependent. It influences the generality of the algorithm in real applications. Also, similar to the existing signal level fusion methods, most of current feature level fusion methods use the iris codes extracted from the original noisy iris captures for fusion. As a result, the fusion result is still influenced by the noise embedded in the feature space.

We note that the algorithms in [20]-[23] are called super resolution in these papers. However, differently from other iris super-resolution methods like [26] and [30], the key step in [20]-[23] is the fusion of information in multiple iris images, rather than the estimation of high-resolution texture from low-resolution observations. Therefore, in this paper, we consider them as information fusion methods.

\section{B. Low Rank Approximation}

Low rank approximation (LRA) is a powerful method to seek the potential noiseless structures of highly-correlated data from noisy observations. Given multiple noisy observations, a basic assumption of LRA is that the potential noiseless data lie in a low rank subspace. Based on this assumption, the LRA of noisy observations is sought as the estimation of noiseless data. Existing LRA algorithms can be categorized into low rank decomposition [26] and low rank matrix factorization [27]-[29], [31]-[35]. Low rank decomposition represents the original noisy observations as the sum of a low rank component and an error component. The low rank component estimates the potential noiseless data, while the error component models the noise which is usually assumed to be spatially sparse. The representative algorithm of low rank decomposition is robust principal component analysis (RPCA) [26]. Let $\boldsymbol{Y} \in \mathbb{R}^{m \times n}$ be a data matrix with $n$ observations (each column is an observation). RPCA seeks the low rank approximation of $\boldsymbol{Y}$ as follows:

$$
\underset{\boldsymbol{A}, \boldsymbol{E}}{\arg \min }\|\boldsymbol{A}\|_{*}+\lambda\|\boldsymbol{E}\|_{1} \quad \text { s.t. } \boldsymbol{Y}=\boldsymbol{A}+\boldsymbol{E}
$$

where $\boldsymbol{A} \in \mathbb{R}^{m \times n}$ is the low rank component and $\boldsymbol{E} \in \mathbb{R}^{m \times n}$ is the error component. $\|\bullet\|_{*}$ is the nuclear norm which calculates the sum of the singular values of $\bullet$. Minimizing $\|\boldsymbol{A}\|_{*}$ leads to a minimization on the rank of $\boldsymbol{A}$. $\|\bullet\|_{1}$ calculates the sum of the absolute value of all the elements in $\bullet$.
Minimizing $\|\boldsymbol{E}\|_{1}$ induces sparsity in $\boldsymbol{E}$. The main advantage of RPCA is its convexity. The convexity enables to solve Eqn. 1 by tractable convex optimization. However, the algorithms to solve Eqn. 1 often require to compute a singular value decomposition (SVD) in each iteration. It is computationally expensive.

Different from low rank decomposition minimizing the nuclear norm to seek a low rank solution, low rank matrix factorization approximates the observation matrix $\boldsymbol{Y}$ by the product of two low rank matrix. It is formulated as follows:

$$
\underset{\boldsymbol{U}, \boldsymbol{V}}{\arg \min }\left\|\boldsymbol{W} \otimes\left(\boldsymbol{Y}-\boldsymbol{U} \boldsymbol{V}^{\mathrm{T}}\right)\right\|
$$

where $\boldsymbol{U} \in \mathbb{R}^{m \times r}$ and $\boldsymbol{V} \in \mathbb{R}^{n \times r}$ are two low rank matrix $(r \ll m, n), \otimes$ is element-wise multiplication operator, and $\boldsymbol{W} \in\{0,1\}^{m \times n}$ is a binary weight matrix indicating missing entries in $\boldsymbol{Y}$. An element in $\boldsymbol{W}$ is assigned 0 if the corresponding entry in $\boldsymbol{Y}$ is missing, otherwise it is assigned 1. Since both $\boldsymbol{U}$ and $\boldsymbol{V}^{\mathrm{T}}$ are low rank matrix, their product is still a low rank matrix and it forms the LRA of $\boldsymbol{Y}$.

Although the solution of Eqn. 2 is not unique and it is also dependent on the structure of $\boldsymbol{W}$, many researches have shown that effective solutions can be computed with the norm in Eqn. 2 being Frobenious norm [28], [29], [31], [32], $\ell_{1}$-norm [27], [33], rotational invariant $R_{1}$-norm [34], or maximizing the $\ell_{1}$ dispersion of $\boldsymbol{Y}$ (i.e. seeking $\boldsymbol{U}$ that maximizes $\left.\left\|\boldsymbol{U}^{\mathrm{T}} \boldsymbol{Y}\right\|_{1}\right)$ [35]. Especially, in recent research, Meng et al. [27] demonstrate that with $\ell_{1}$-norm, Eqn. 2 can be efficiently solved by a cyclic weighted median filtering algorithm. As reported in [27], this method achieves a more robust reconstruction of noiseless signals in comparison to RPCA and some other representative algorithms of low rank matrix factorization.

However, compared to low rank decomposition, low rank matrix factorization does no explicitly formulate the noise component (i.e. $\boldsymbol{E}$ in Eqn. 1). The noise elements can be indicated in $\boldsymbol{W}$, but such weighting scheme heavily relies on the prior of noise positions which are sometimes unavailable in real applications. As a result, the noise in the observations always influences the estimation of $\boldsymbol{U}$ and $\boldsymbol{V}$ in the procedure of solving Eqn. 2, although such influence can be mitigated by using some more robust norms, like the above mentioned $\ell_{1}$-norm, rotational invariant $R_{1}$-norm, and $\ell_{1}$ dispersion of $\boldsymbol{Y}$.

\section{The Proposed Method}

In this section, we present the proposed signal level fusion method for less constrained iris recognition. Firstly, we describe the proposed sparse-error low rank matrix factorization (SE-LRMF) model to seek a low rank approximation. Given multiple iris captures, SE-LRMF decomposes them into a low rank component expressing the potential noiseless images and an error component estimating the noise. Then, we demonstrate our signal level fusion method based on the result of SE-LRMF. It consists of a low rank mean (LRM) fusion method using the low rank component and an error weighted mean (EWM) fusion method employing the error component. Finally, we present the code level combination of the results of LRM and EWM. 


\section{A. Sparse-Error Low Rank Matrix Factorization (SE-LRMF)}

Given multiple noisy captures of the same eye, we assume that the potential noiseless images lie in a low rank subspace, due to the inherent stability of iris patterns. Similar to the notations in section II-B, we use $\boldsymbol{Y} \in \mathbb{R}^{m \times n}$ to denote a data matrix with each of its column being an unwrapped iris image. We use $\boldsymbol{U} \in \mathbb{R}^{m \times r}$ and $\boldsymbol{V} \in \mathbb{R}^{n \times r}$ to denote the two matrix forming the factorization of $\boldsymbol{Y}$, and we use $\boldsymbol{E} \in \mathbb{R}^{m \times n}$ to denote the error component which models the noise in the iris images. The proposed SE-LRMF model is formulated as follows:

$$
\underset{\boldsymbol{U}, \boldsymbol{V}, \boldsymbol{E}}{\arg \min } \frac{1}{2}\left\|\boldsymbol{Y}-\boldsymbol{U} \boldsymbol{V}^{\mathrm{T}}-\boldsymbol{E}\right\|_{F}^{2}+\lambda\|\boldsymbol{E}\|_{1}
$$

where $\lambda$ is a penalty parameter. Eqn. 3 includes two terms. The first term is a reconstruction fidelity term. It represents the noisy iris captures by the sum of the noiseless images (low rank component $\boldsymbol{U} \boldsymbol{V}^{\mathrm{T}}$ ) and noise (error component $\boldsymbol{E}$ ). The second term is a sparsity constraint on the error component. Following RPCA [26], we assume that the noise is spatially sparse, and we use $\ell_{1}$-norm to induce sparsity. The model has two parameters: $r \ll m, n$ is one of the dimensions of $\boldsymbol{U}$ and $\boldsymbol{V}$, controlling the rank of approximation; $\lambda$ is a scalar balancing between the reconstruction error and the sparsity.

Different from traditional low rank matrix factorization models in Eqn. 2, SE-LRMF explicitly formulates the noise to suppress its influence. Also, compared to Eqn. 2, Eqn. 3 does not include $\boldsymbol{W}$ which is the weight matrix indicating missing entries. The reason is three-fold. First, although we can construct $\boldsymbol{W}$ based on the results of iris segmentation, the segmentation errors will lead to inaccurate entries. Even with an accurate localization of iris, pupil and eyelids, false detections on reflection and shadow still induce inaccuracy. Second, including $\boldsymbol{W}$ increases the complexity of the objective function, and it is possible to induce more computational load. Third, experimentally, we find that the error component $\boldsymbol{E}$ and the sparsity constraint on it are powerful enough to model the noise and mitigate its influence on the estimation of $\boldsymbol{U}$ and $\boldsymbol{V}$.

Eqn. 3 is not convex in $\boldsymbol{U}, \boldsymbol{V}$ and $\boldsymbol{E}$, but it is convex in one of them with the other two fixed. Therefore, we seek a local minimum via an alternating scheme. It leads to a $\boldsymbol{U}$, $\boldsymbol{V}$-subproblem and an $\boldsymbol{E}$-subproblem.

$\boldsymbol{U}, \boldsymbol{V}$-Subproblem: Let $\widetilde{\boldsymbol{Y}}=\boldsymbol{Y}-\boldsymbol{E}$. With error component $\boldsymbol{E}$ fixed, minimizing Eqn. 3 leads to the following problem:

$$
\underset{\boldsymbol{U}, \boldsymbol{V}}{\arg \min }\left\|\widetilde{\boldsymbol{Y}}-\boldsymbol{U} \boldsymbol{V}^{\mathrm{T}}\right\|_{F}^{2}
$$

The solution of Eqn. 4 is not unique. By fixing one of $\boldsymbol{U}$ and $\boldsymbol{V}$ and setting the partial derivative of the objective function with respect to the other one to zero, we can descend the objective function by updating $\boldsymbol{U}$ and $\boldsymbol{V}$ as follows:

$$
\begin{aligned}
& \boldsymbol{V}=\widetilde{\boldsymbol{Y}}^{\mathrm{T}} \boldsymbol{U}\left(\boldsymbol{U}^{\mathrm{T}} \boldsymbol{U}\right)^{-1} \\
& \boldsymbol{U}=\widetilde{\boldsymbol{Y}} \boldsymbol{V}\left(\boldsymbol{V}^{\mathrm{T}} \boldsymbol{V}\right)^{-1}
\end{aligned}
$$

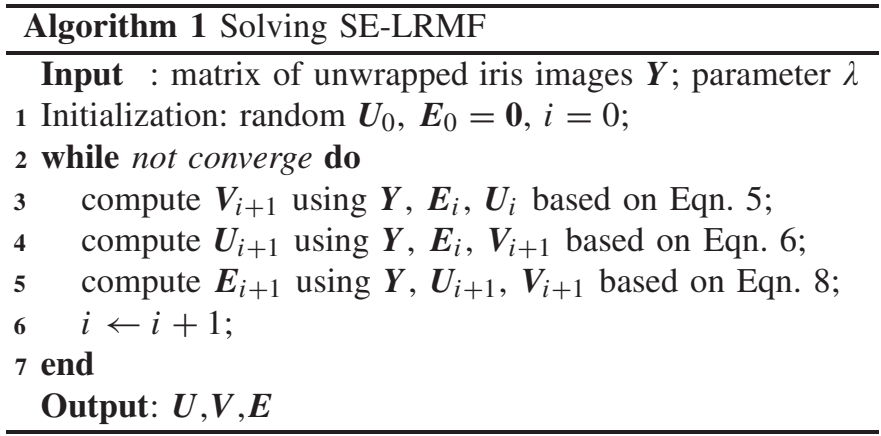

$\boldsymbol{E}$-Subproblem: Let $\widehat{\boldsymbol{Y}}=\boldsymbol{Y}-\boldsymbol{U} \boldsymbol{V}^{\mathrm{T}}$. With $\boldsymbol{U}$ and $\boldsymbol{V}$ fixed, minimizing Eqn. 3 leads to the following problem:

$$
\underset{\boldsymbol{E}}{\arg \min } \frac{1}{2}\|\widehat{\boldsymbol{Y}}-\boldsymbol{E}\|_{F}^{2}+\lambda\|\boldsymbol{E}\|_{1}
$$

According to [36], the solution of Eqn. 7 can be obtained by performing soft-thresholding operation on $\widehat{\boldsymbol{Y}}$ :

$$
\boldsymbol{E}=\operatorname{sign}(\widehat{\boldsymbol{Y}}) \max (|\widehat{\boldsymbol{Y}}|-\lambda, 0)
$$

where sign $(\bullet)$ is the sign function. All the operations in Eqn. 8 are element-wise.

With the parameter $\lambda$ fixed, alternating between $\boldsymbol{U}$, $\boldsymbol{V}$-subproblem and $\boldsymbol{E}$-subproblem leads to a sequence of monotonically decreasing objective function values. Therefore, it will converge to a local minimum.

It can be seen that in such an alternating scheme, $\boldsymbol{U}$ and $\boldsymbol{V}$ forming the low rank component are computed based on noise-eliminated signals $\widetilde{\boldsymbol{Y}}=\boldsymbol{Y}-\boldsymbol{E}$. In other words, with the explicitly formulated error component $\boldsymbol{E}$, the proposed SE-LRMF is able to suppress the influence of noise on low rank matrix factorization without relying on any specific robust norms or weight matrix based on the priors of noise.

In our implementation, the order of alternation is Eqn. 5, Eqn. 6, Eqn. 8. Accordingly, we need to initialize $\boldsymbol{U}$ and $\boldsymbol{E}$ in the first iteration. In our experiment, we find that although Eqn. 3 has multiple local minimum and the solution depends on the starting point, a simple random initialization of $\boldsymbol{U}$ as used in [27] is able to achieve a generally good performance. $\boldsymbol{E}$ is initialized to be a matrix of zeros. We summarize the whole algorithm to solve Eqn. 3 in Algorithm 1.

After obtaining $\boldsymbol{U}, \boldsymbol{V}$ and $\boldsymbol{E}$, we construct a matrix $\boldsymbol{A}=\boldsymbol{U} \boldsymbol{V}^{\mathrm{T}}$ to be the low rank component considered as noiseless iris images, and we directly use the error component $\boldsymbol{E}$ as the estimated noise. Some example results of SE-LRMF is shown in Fig. 2(a) to Fig. 2(c). It can be seen that given the noisy iris captures in Fig. 2(a), the low rank components in Fig. 2(b) mainly include the potential stable structure of the iris, while the error component in Fig. 2(c) accurately models noise like reflections, eyelids and eyelashes. We note that part of eyelids and eyelashes are still contained in the low rank component. This is because the pixels in some regions are always occupied by noise, and we lack the noiseless information for these regions. Also, we find that some clear iris texture is possible to be detected as noise. An example is the second image from the top. It can be seen that in this image, 


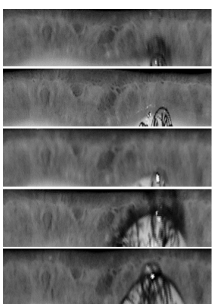

(a)

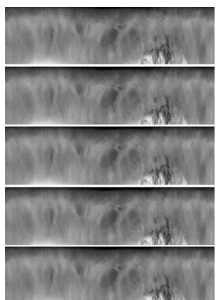

(b)

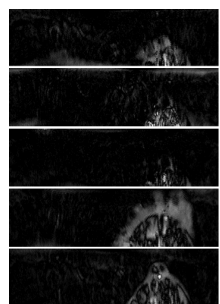

(c)

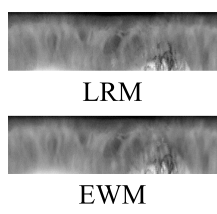

(d)
Fig. 2. Examples of the results of SE-LRMF based signal level fusion. (a) The original unwrapped iris captures from the same eye; (b) the low rank component (noiseless iris images) of the images in (a) sought by SE-LRMF; (c) the error component (noise) of the images in (a) sought by SE-LRMF (d) the fusion result using low rank mean (LRM) fusion and error weighted mean (EWM) fusion.

some clear iris textures in the middle part at the horizontal direction are included in the error component. The reason is that these local textures in this image are visually different from the textures in other images at the same position. We note that this leads to information loss. We will handle this problem at the signal level fusion stage.

\section{B. Signal Level Fusion}

Given multiple noisy iris captures of the same eye, we can seek the a low rank component $\boldsymbol{A}$ as the noiseless images and an error component $\boldsymbol{E}$ as the noise using the SE-LRMF model proposed in section III-A. In this subsection, we utilize $\boldsymbol{A}$ and $\boldsymbol{E}$ individually for signal level fusion. It leads to a low rank mean (LRM) fusion and an error weighted mean (EWM) fusion.

1) Low Rank Mean (LRM) Fusion: Since $\boldsymbol{A}$ is viewed as a matrix of noiseless iris images, we can fuse the images in $\boldsymbol{A}$ directly by mean fusion. We produce a fused image by calculating the mean of all the noiseless images in $\boldsymbol{A}$. This fusion method is named low rank mean, since it is the mean fusion on the low rank component sought by SE-LRMF.

However, simple mean fusion on noiseless component is not enough. We find that although the influence of noise is mitigated in the low rank component $\boldsymbol{A}$, some images in $\boldsymbol{A}$ suffer from texture loss. The reason is that in some cases, some local textures in one image are visually different from the textures in other images at the same position. Consequently, these textures are possible to be excluded from the low rank component $\boldsymbol{A}$ and considered as noise (see the example discussed at the end of section III-A). It results information loss and influence the performance of iris recognition using LRM fusion. Therefore, we propose an error weighted mean (EWM) fusion method. EWM fusion utilizes all the information in the original images. It mitigates the influence of noise by a weighting scheme based on the error component $\boldsymbol{E}$ sought by SE-LRMF.

2) Error Weighted Mean (EWM) Fusion: Recall that we denote the matrix of original iris captures by $\boldsymbol{Y} \in \mathbb{R}^{m \times n}$ with each of its column being an observation. Let $\boldsymbol{y}_{i} \in \mathbb{R}^{m}$ be the $i^{\text {th }}$ column of $\boldsymbol{Y}$. The proposed EWM fusion is formulated as follows:

$$
\boldsymbol{y}^{f}=\omega^{1} \otimes \boldsymbol{y}_{1}+\omega^{2} \otimes \boldsymbol{y}_{2}+\cdots+\omega^{n} \otimes \boldsymbol{y}_{n}
$$

where $\boldsymbol{y}^{f} \in \mathbb{R}^{m}$ denotes the fused image, $\otimes$ is elementwise multiplication operator, $\omega^{i}=\left[\omega_{1}^{i}, \omega_{2}^{i}, \ldots, \omega_{m}^{i}\right]^{\mathrm{T}} \in \mathbb{R}^{m}$ is a vector of weights corresponding to $\boldsymbol{y}_{i}$. The weights in $\omega^{1}, \omega^{2}, \ldots, \omega^{n}$ satisfy that for any arbitrary $j \in[1, m]$, $\sum_{i=1}^{n} \omega_{j}^{i}=1$. It means that the weights on the same position of all the images have a sum of 1 .

Different from the global weighting scheme in the existing weighted mean fusion methods [21], [22], EWM uses a pixelwise weighting scheme. We assign the weights considering the individual reliability of each pixel; the weights of different pixels in the same image can be different. Compared to the existing global weighting method, EWM has a more flexible and finer assignment of the weights. We expect that it is able to better preserve usable information while suppressing noise.

Given Eqn. 9, the key problem is how to compute the weights in $\omega^{1}, \omega^{2}, \ldots, \omega^{n}$. In this paper, we compute these weights based on the error component $\boldsymbol{E}$ obtained by SE-LRMF model. The idea is straightforward: we consider $\boldsymbol{E}$ as the estimation of noise; for a pixel, the higher the noise level, the lower the reliability of the information in this pixel; hence, the weights should be inversely proportional to $|\boldsymbol{E}| \in \mathbb{R}^{m \times n}$. Based on this idea, we calculate the weights as follows.

Firstly, we normalize the elements in $|\boldsymbol{E}|$ to between 0 and 1 by:

$$
\boldsymbol{E}^{\prime}=\frac{|\boldsymbol{E}|-f_{\min }(|\boldsymbol{E}|)}{f_{\max }(|\boldsymbol{E}|)-f_{\min }(|\boldsymbol{E}|)}
$$

where $\boldsymbol{E}^{\prime}$ denote the normalized matrix of $|\boldsymbol{E}|, f_{\min }(\bullet)$ and $f_{\max }(\bullet)$ find the minimum and maximum value in a matrix, respectively.

Then, we calculate a weight matrix $\boldsymbol{W}^{\prime} \in \mathbb{R}^{m \times n}$ by:

$$
\boldsymbol{W}^{\prime}=\mathbf{1}-\boldsymbol{E}^{\prime}
$$

Thus, the elements in $\boldsymbol{W}^{\prime}$ are inversely proportional to $|\boldsymbol{E}|$.

Finally, we normalize each row of $\boldsymbol{W}^{\prime}$ to have a sum of 1 . We use the columns of the normalized $\boldsymbol{W}^{\prime}$ as $\omega^{1}, \omega^{2}, \ldots, \omega^{n}$. The reason is that each column of $\boldsymbol{E}$ corresponds to the estimated noise in an iris capture, so each column of the normalized $\boldsymbol{W}^{\prime}$ can be considered as the weights corresponding to each image.

3) Iris Mask Production: The final problem in signal level fusion is how to produce an iris mask for the fused image. This iris mask indicates the detected noise like specular reflection and eyelids. In this paper, we adopt a simple AND rule: given a set of iris captures, a pixel is masked as noise if it is detected as noise in the iris segmentation phase of any individual iris captures. We note that such a scheme is possible to lead to information loss, since it is possible to eliminate the pixels successfully reconstructed in the low rank component. A better solution is to seek a threshold based on the times a position is detected as noise in all the iris captures. However, we experimentally find that the AND rule we use is sufficient to obtain a well performance. Also, it avoids the need for selection of a threshold which might be data dependent.

Example results of LRM and EWM are shown in Fig. 2(d). We would like to note that despite of a similar visual appearance, the results of these two methods contain the information sought from different aspects: LRM eliminates all the estimated noise at a cost of information loss; 
EWM utilizes all the information in the original iris captures while suppressing the estimated noise.

\section{Code Level Combination}

The result of either LRM or EWM fusion is not fully noisefree. For LRM, it is possible to fail to estimate the noiseless information when a position is consistently occupied by noise in most of the images. As for EWM, it utilizes the original iris captures for fusion. The weighting scheme of EWM is able to mitigate the noise, but not fully eliminate it. Therefore, we propose a code level combination method to further suppress the noise in the results of LRM and EWM.

This code level combination method is based on the inherent stability of iris patterns: assuming that we have some fully noise-free iris captures of the same eye, the iris codes extracted from these captures should be highly consistent. Based on this, we can seek more reliable bits in iris codes via the bit consistency between the iris codes extracted from the LRM and EWM results. In other words, a bit is considered more reliable if it is consistent between the iris codes extracted from LRM and EWM results. By only preserving these more reliable bits for iris matching stage, we expect that an improved performance can be achieved. Essentially, the idea is similar to the iris weight map method [7], [8], [15].

Specifically, given multiple noisy iris captures of the same eye, we obtain two images via the proposed signal level fusion, one using LRM and the other one using EWM. We extract two iris codes from the two images. We fuse the two iris codes by only preserving the bits that are consistent between them. This can be simply implemented by using either one of the two iris codes as the final iris code, and eliminating the inconsistent bits between the two iris codes in the corresponding iris mask.

\section{EXPERIMENT}

In this section, we present the results of experimental analysis for the proposed signal level fusion method. First, we introduce our experiment setting, including datasets, fusion setting, parameter setting and performance evaluation (section IV-A). Then, with the low rank approximation method fixed, we analyse the effect of each individual signal level fusion step, including low rank mean fusion, error weighted mean fusion and code level combination (section IV-B). Next, with the signal level fusion steps fixed, we study the performance of the propose SE-LRMF model for low rank approximation (section IV-C). We compare the performance of SE-LRMF with representative low rank approximation algorithms. After that, we compare the proposed SE-LRMF based signal level fusion with existing methods, including no-fusion (section IV-D), signal level fusion (section IV-E), score level fusion (section IV-F) and other recent iris recognition algorithms (section IV-G). Finally, we analyse the influence of noise level on the performance of our algorithm (section IV-H).

\section{A. Experiment Setting}

1) Datasets: We conduct the experiments on three benchmark datasets: ND-iris-0405 [37], CAISA.v4 distance [38] and UBIRIS.v2 [12]. The three datasets cover the data captured in varying less constrained environments. ND-iris-0405 (also referred as ND0405 in this paper) is a large-scale dataset captured in NIR wavelength and at a close distance. Many real-world conditions occur in this dataset, leading to degradations in the captures, including blurring, eyelids occlusion, specular reflection, off-angle, etc. Also, some subjects wear soft contact lenses which cause distortion on iris textures. The current version of this dataset includes 64,982 iris images from 712 eyes. In our experiment, we use a subset with the first 15 images of each eye. For the eyes with less than 15 images, all the iris images are used. Furthermore, we eliminate 82 images with too poor quality from the subset (these images have too small iris region due to factors like heavy eyelids occlusion and large off-angle). It leads to a ND-iris-0405 subset consisting of 9811 images from 712 eyes to use in the experiment. This subset represents the iris data with a relatively lower noise level. The iris images in this subset are captured at a noiseless wavelength (NIR) and a close distance, hence with good resolution and clear iris texture, but suffering from the noise due to real-world conditions.

CASIA.v4 distance (also referred as CASIA4 in this paper) is a dataset of images captured at a distance under NIR wavelength. The stand-off distance is around 3 meters. It consists of 142 subjects. The images include most of facial features and patterns. The eye region of most images suffers from blinking, eyelids occlusion, specular reflection and motion blur. In our experiment, we detect left and right eye regions using classical Viola-Jones object detector [39]. We correct false detections manually, and we eliminate 97 eye regions with too poor quality (most of these eye regions are completely covered by specular reflection). It results a subset with 5037 iris images from 284 eyes to use in the experiment. This dataset represents the iris data with a medium noise level. The images are captured at NIR wavelength but at a distance. As a result, the iris region has a low resolution; the iris texture is visually less clear; the noise in this dataset is heavier than that in ND-iris-0405.

UBIRIS.v2 (also referred as UBIRIS2 in this paper) is a colour iris dataset captured with dynamic lighting conditions. The stand-off distance is between 3 and 8 meters. The images are influenced by specular reflection across the eye region, eyelids occlusion, off-angle and blurring. The whole dataset consists of 11, 102 images from 261 subjects. In our experiment, we use a subset with 1000 images from 171 eyes. This subset was released for NICE II contest [11]. We think it is reasonable to use this subset as a representative set for the data in the whole UBIRIS2 dataset, because of its good generality. This subset was released for a public contest, and it is widely used in many following researches like [14]-[16] and [40]. Also, due to its good generality, using this subset enables to roughly compare the performance of algorithms in relevant literature. For UBIRIS.v2, we operate on the red channel of RGB colour space. This dataset represents the iris data with a relatively higher noise level. The images are captured not only at a distance, but also in a noisy wavelength (colour). We show some examples of the images in the three datasets in Fig. 3. 


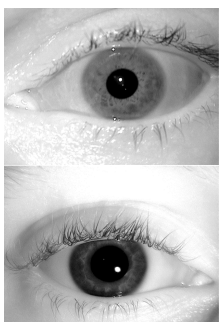

(a)

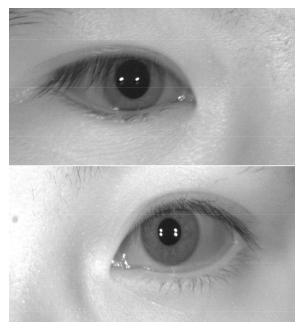

(b)

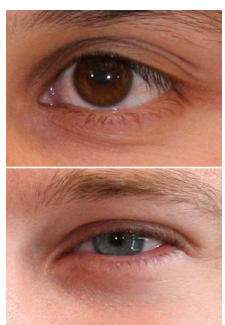

(c)
Fig. 3. Example images of the dataset we use. (a) ND-iris-0405; (b) CASIA.v4 distance; (c) UBIRIS.v2.

For all the datasets, we segment and unwrap the iris region using the algorithm in [41]. We correct the failed segmentations manually. The size of unwrapped iris image is set to $100 \times 360$. We divide the unwrapped iris image by a factor of 255 to normalize the intensity to $[0,1]$. We use 1-D log-Gabor filter [42] to extract iris codes.

2) Fusion Setting: Given the above datasets, we need to construct gallery and probe sets for experimental analysis of information fusion methods. In this paper, we simulate the scenario where both gallery and probe images are captured in less constrained environment, so both of them suffer from the influence of noise. Accordingly, we construct both gallery and probe sets by fusing the information in multiple noisy captures. We note that some researches like [20] and [21] directly use high quality still iris images as gallery without fusion. We do not follow this scenario because it is difficult to obtain high quality still iris images to construct the gallery in some applications like surveillance or mobile device-based capture. We simulate a scenario which has less requirements on the iris image quality in both registration and iris matching phases. Under such scenario, most existing feature level fusion methods [22]-[24], [27] are less applicable, since these methods need high quality iris images to learn the relationship between low resolution and high resolution features. Thus, we consider signal level fusion and score level fusion in our experiment.

Based on the above simulated scenario, for signal level fusion, we construct the gallery and probe sets as follows. For a specific eye, we fuse the first 5 images to produce a single gallery iris code. Then, from the remaining images of this eye, we randomly select 5 images and fuse them to produce a probe iris code. To produce multiple probe iris codes, the above random selection and fusion based on the remaining images are performed multiple times. It simulates the case that in the iris matching phase, the captured images can be different at each time of matching. If an eye has less than 5 images, we fuse all the images to produce the gallery iris code, and there are no probe iris codes. If an eye has less than 10 images, we fuse the first 5 images to produce the gallery iris code, and we fuse all the remaining images to produce a probe iris code without random selection. The number of images for fusion (5) is chosen so that we have enough information for fusion, while we can produce a sufficient number of probe iris codes as well.

For score level fusion, the fusion setting is similar. The difference is that, given a set of iris captures, instead of fusing
TABLE I

Parameter Setting on Each Dataset

\begin{tabular}{|c|c|c|c|c|}
\hline Dataset & $r$ & $\lambda$ & wavelength & sigmaOnf \\
\hline ND0405 & 1 & 0.01 & 35 & 0.49 \\
\hline CASIA4 & 1 & 0.01 & 19 & 0.48 \\
\hline UBIRIS2 & 1 & 0.01 & 40 & 0.38 \\
\hline
\end{tabular}

them and producing one iris code, we extract iris codes from each individual iris capture to produce an iris code set. In the matching phase, given a gallery iris code set and a probe iris code set, the final matching score is calculated by fusing the matching scores between the iris codes in the probe and gallery iris code sets.

3) Parameter Setting and Performance Evaluation: We use exclusive data for parameter setting and performance evaluation. For ND-iris-0405 dataset, we use the first 20 eyes to tune the parameters, and we use the remaining 692 eyes to evaluate the performance. For CASIA.v4 distance dataset, we use the first 20 eyes to tune the parameters, and we use the remaining 264 eyes to evaluate the performance. For UBIRIS.v2 dataset, we use the first 19 eyes to tune the parameters, and we use the remaining 152 eyes to evaluate the performance.

Considering parameter setting, there are 4 parameters to be determined: $r$ is the rank of low rank approximation in SE-LRMF model; $\lambda$ in Eqn. 3 is a parameter controlling the trade-off between the reconstruction error and the sparsity of noise; wavelength and sigmaOnf are 1-D log-Gabor parameters. Based on the above described data for parameter tuning, we report the setting of these parameters in Tab. I.

For performance evaluation of signal level fusion, the above settings of fusion and performance evaluation data lead to the following gallery and probe sets: 692 gallery iris codes and 6200 probe iris codes for ND-iris-0405; 264 gallery iris codes and 5114 probe iris codes for CASIA.v4 distance; 152 gallery iris codes and 606 probe iris codes for UBIRIS.v2. As for score level fusion, the number of fused intra-class and interclass matching scores is exactly the same as signal level fusion.

\section{B. Analysis of Each Signal Level Fusion Step}

As described in section III-B, given the low rank and error components produced by SE-LRMF, the proposed method includes three fusion steps: low rank mean (LRM) fusion, error weighted mean (EWM) fusion and code level combination (CLC). In this subsection, we analyse the effect of each individual fusion step. We use mean fusion (MF) as the baseline, because EWM is essentially an improved mean fusion. We show the CMC and ROC curves of MF, LRM, EWM, CLC in Fig. 4.

We have two main observations from Fig. 4. First, EWM achieves a generally better performance than MF. This result illustrates that the proposed EWM can better mitigate the noise in the iris captures compared to MF. Second, it can be seen that although the performance of LRM and EWM varies on different datasets, CLC consistently leads to improved CMC and ROC curves compared to either LRM or EWM on all the datasets. This observation suggests that with SE-LRMF for 


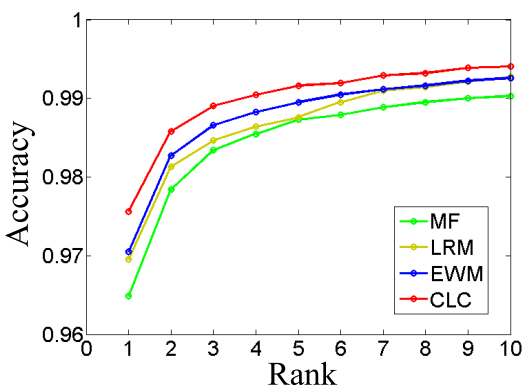

(a)

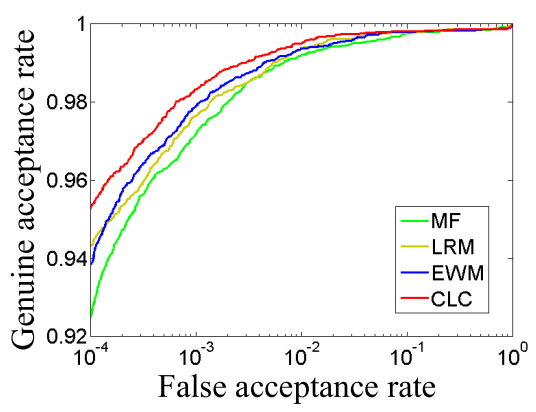

(d)

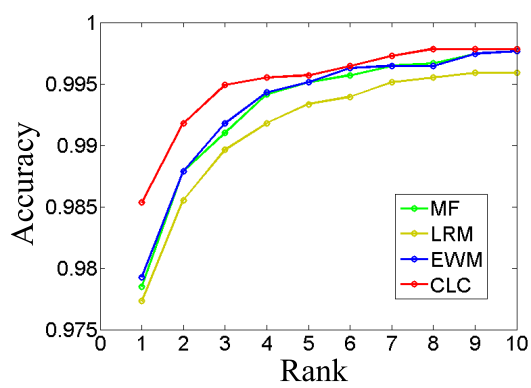

(b)

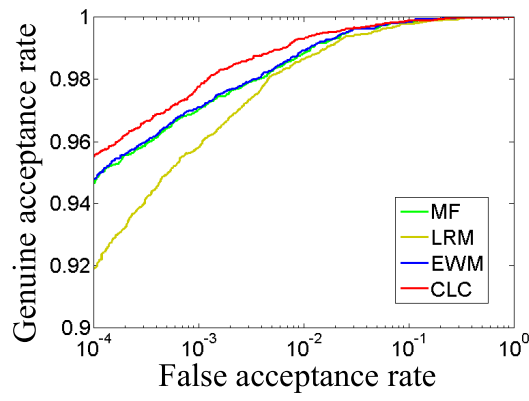

(e)

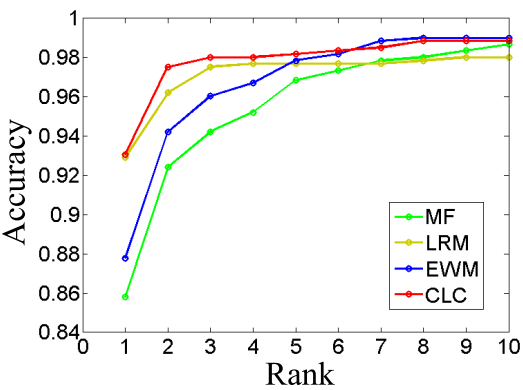

(c)

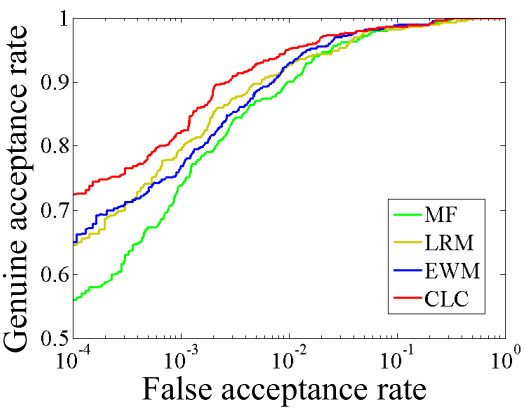

(f)

Fig. 4. The CMC and ROC curves to analyse each signal level fusion step. (a) CMC, ND0405. (b) CMC, CASIA4. (c) CMC, UBIRIS2. (d) ROC, ND0405. (e) ROC, CASIA4. (f) ROC, UBIRIS2.

low rank approximation, CLC is able to achieve an improved and more stable performance in comparison to LRM and EWM. It validates the effectiveness of the proposed code level combination.

\section{Comparison Between SE-LRMF and Existing Low Rank Approximation Algorithms}

In this subsection, we make comparisons between the proposed SE-LRMF model and existing low rank approximation (LRA) algorithms to study the effectiveness of SE-LRMF in the proposed framework of signal level fusion. We make the comparison by: (1) fixing the fusion steps (LRM, EWM, CLC); (2) varying the algorithm of LRA to produce the low rank and error components used by the fusion steps.

As described in section II-B, existing LRA algorithms can be categorized into low rank decomposition and low rank matrix factorization. Accordingly, we make the comparison with a representative low rank decomposition algorithm and a representative low rank matrix factorization algorithm. In terms of low rank decomposition, we make the comparison with robust principal component analysis (RPCA) in [26], since RPCA is currently one of the most widely used algorithms for low rank decomposition. As for low rank matrix factorization, we compare with the cyclic weight median algorithm with $\ell_{1}$ error term proposed in [27] (referred as L1-LRMF). As reported in [27], this algorithm outperforms most existing LRA algorithms in the task of reconstructing noiseless images from multiple corrupted observations. To eliminate the influence of CLC and have an insight on the effect of the low rank and error components produced by different LRA algorithms, we report the performance of LRM, EWM and CLC with different LRA algorithms in Fig. 5.

We have several observations from the results in Fig. 5. First, considering the results of LRM, we find that the proposed SE-LRMF achieves a generally better performance in comparison to RPCA and L1-LRMF. Specifically, on ND-iris-0405 dataset, LRM with SE-LRMF achieves comparable identification and verification performance to LRM with L1-LRMF, and the the performance is better than RPCA. On CASIA.v4 distance and UBIRIS.v2 datasets, LRM with SE-LRMF outperforms LRM with RPCA and L1-LRMF in both identification and verification. Moreover, the performance of LRM with SE-LRMF is more stable. It can be seen that LRM with L1-LRMF performs better than LRM with RPCA on ND-iris-0405 and UBIRIS.v2 datasets, while LRM with RPCA is better than LRM with L1-LRMF on CASIA.v4 distance dataset. In contrast, LRM with SE-LRMF achieves a more stable performance at a top level on all the datasets. This observation suggests that, for iris recognition using LRM fusion, the low rank components sought by SE-LRMF are able to achieve a more stable and top-level performance, compared to the low rank components sought by RPCA and L1-LRMF.

Second, generally, we find that EWM with different LRA algorithms have similar performance. It means that varying the LRA algorithm has little influence on the result of EWM. We think the reason is that although different LRA algorithms lead to varying estimations on low rank components, these LRA algorithms have similar results on error components. Therefore, EWM with different LRA algorithms has similar performance. The only exception is ND-iris-0405 where EWM with SE-LRMF and L1-LRMF perform better than EWM with RPCA. Considering that ND-iris-0405 is captured at a close 


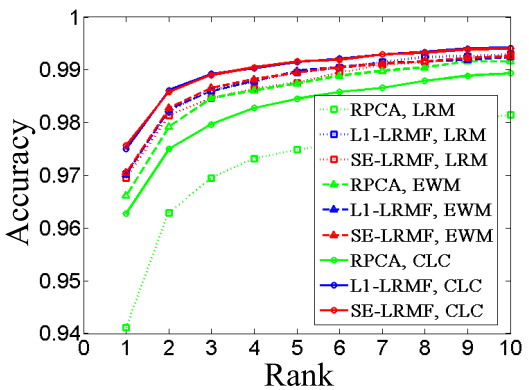

(a)

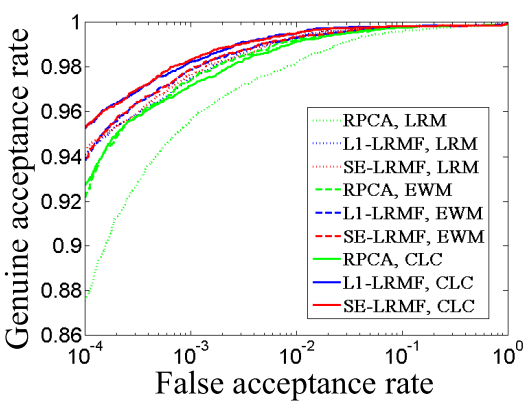

(d)

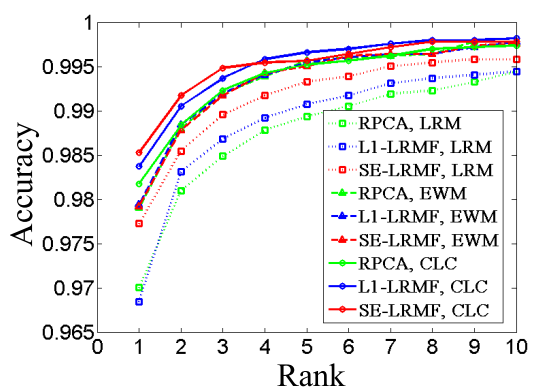

(b)

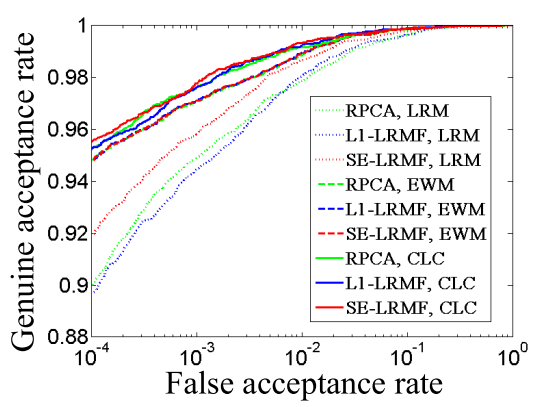

(e)

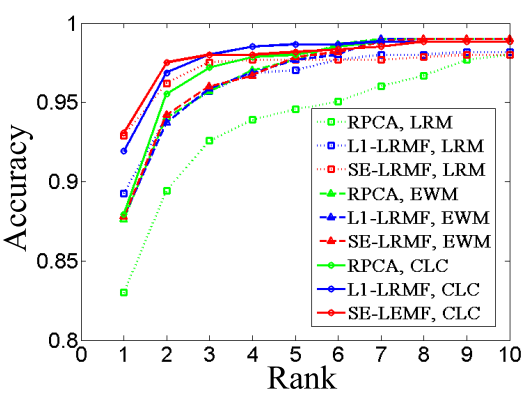

(c)

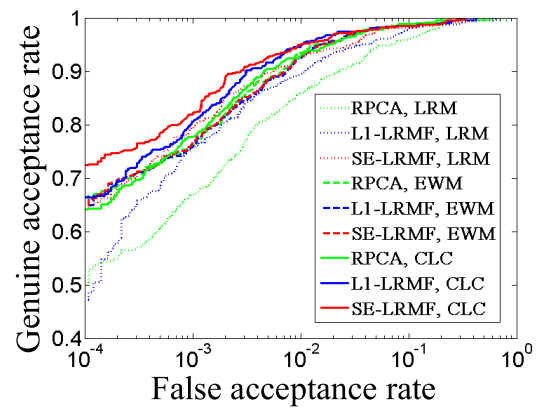

(f)

Fig. 5. The CMC and ROC curves for the comparison between SE-LRMF and existing low rank approximation models. (a) CMC, ND0405. (b) CMC, CASIA4. (c) CMC, UBIRIS2. (d) ROC, ND0405. (e) ROC, CASIA4. (f) ROC, UBIRIS2.

distance, we think this observation may suggest that EWM with SE-LRMF and L1-LRMF are preferable for this type of iris data. When the capturing distance grows, EWM with different LRA algorithms have little difference.

Third, we find that given the similar performance of EWM, the performance variation of CLC with different LRA algorithms is not as large as that of LRM with different LRA algorithms. In our opinion, this observation validates the effectiveness of CLC: it further eliminates the unreliable bits in LRM results, hence different LRA algorithms have similar performance after CLC. However, we can find that CLC with SE-LRMF still has a top performance in comparison to CLC with RPCA and L1-LRMF. In terms of identification, on ND-iris-0405 dataset, CLC with SE-LRMF achieves a comparable rank 1 recognition accuracy to CLC with L1-LRMF, and this performance is better than CLC with RPCA. On CASIA.v4 distance and UBIRIS.v2 datasets, the rank 1 recognition accuracy of CLC with SE-LRMF is higher than that of CLC with RPCA and L1-LRMF. As for verification, on ND-iris-0405 dataset, CLC with SE-LRMF achieves a comparable ROC curve to CLC with L1-LRMF, and this performance is better than CLC with RPCA. On CASIA.v4 distance dataset, the ROC curve of CLC with SE-LRMF is slightly better than that of CLC with RPCA and L1-LRMF. On UBIRIS.v2 dataset, CLC with SE-LRMF also leads a better ROC curve. Considering the noise level of all the datasets $($ ND0405 (close + NIR) $<$ CAISA4 (distant + NIR) $<$ UBIRIS2 (distant + colour)), we can say that CLC with SE-LRMF performs better for the data with relatively heavier noise, while it is still able to achieve a top performance on the data with less noise.
Four, we find that CLC does not always lead to an improved performance over LRM and EWM. On ND-iris-0405 dataset, CLC with RPCA has a lower rank 1 recognition accuracy than EWM with RPCA. Also, on ND-iris-0405 and UBIRIS.v2 datasets, the ROC curves of CLC with RPCA and EWM with RPCA are very similar. In our opinion, a possible reason is that on these two datasets, the result of LRM with RPCA contains a high level of noise. The noise level is so high that it influences the result of CLC: too many noiseless bits in EWM results are excluded due to their inconsistency with the noisy bits in LRM results. In other words, the result of CLC is biased due to noise.

We conduct an experiment to gain a deeper insight into the above bias effect. In this experiment, we measure the noise level in LRM and EWM results by the percentage of inconsistent bits between the iris codes extracted from LRM and EWM results. A higher percentage of inconsistent bits means that at least one of the LRM and EWM results has a high noise level, since the iris codes extracted from LRM and EWM results should be highly consistent if both LRM and EWM results are low-noise or noise-free, due to the inherent stability of iris pattern. The detailed experiment is as follows. Given the fusion setting in section IV-A, we have multiple image sets for fusion to produce gallery and probe iris codes. Given a specific image set and a LRA algorithm, we produce a LRM fused image and an EWM fused image, and we measure the percentage of inconsistent bits between the iris codes extracted from the two LRM and EWM fused images. For a specific LRA algorithm, we use the mean percentage of inconsistent bits calculated using all the image sets as the estimation of the noise level in the fusion results of this 


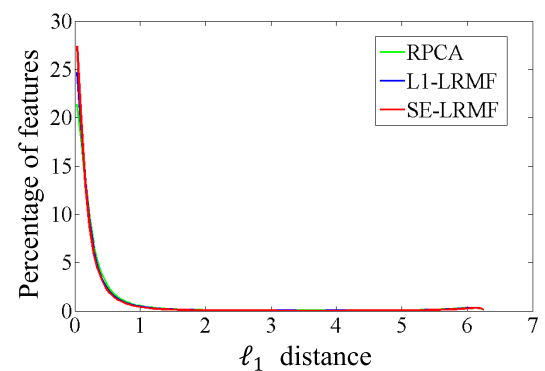

(a)

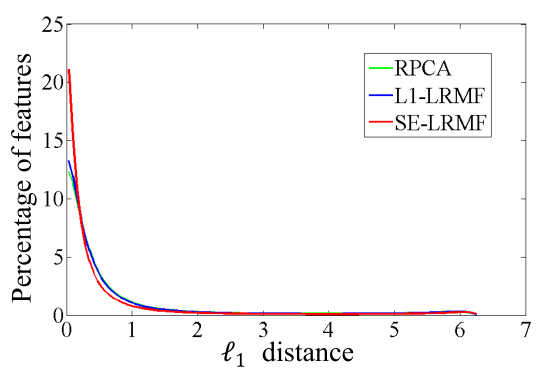

(b)

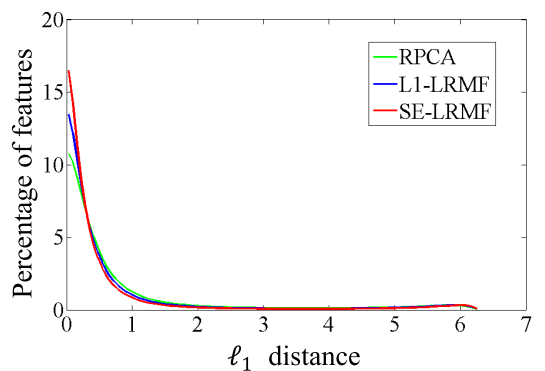

(c)

Fig. 6. The distribution of $\ell_{1}$ distances between the raw feature values extracted from LRM and EWM results. (a) ND0405. (b) CASIA4. (c) UBIRIS2.

TABLE II

Percentage of InCONSISTENT Bits Between LRM AND EWM FUSION RESULTS

\begin{tabular}{|c|c|c|c|}
\hline Dataset & RPCA & L1-LRMF & SE-LRMF \\
\hline ND0405 & $9.74 \%$ & $8.88 \%$ & $8.29 \%$ \\
\hline CASIA4 & $18.02 \%$ & $17.36 \%$ & $14.04 \%$ \\
\hline UBIRIS2 & $23.47 \%$ & $20.71 \%$ & $17.61 \%$ \\
\hline
\end{tabular}

LRA algorithm (note that we do no distinguish gallery and probe). In Tab. II, we report the percentage of inconsistent bits measured using RPCA, L1-LRMF and SE-LRMF on the three datasets.

Investigating on the results in Tab. II, we find that the percentage of inconsistent bits increases with the noise level of datasets (ND0405 < CAISA4 < UBIRIS2). It is consistent with our above analysis to use the percentage of inconsistent bits to measure the noise level. Also, it is very clear that RPCA have the highest percentage of inconsistent bits among the three LRA algorithms. It means that the fusion results with RPCA contain relatively heavier noise. Consequently, the result of CLC with RPCA is more likely to be biased due to the noise in the LRM and EWM fused images. This explains our above experimental observation four. Moreover, we can find that SE-LRMF has the lowest percentage of inconsistent bits among the three LRA algorithms on all the three datasets. This observation demonstrates that the LRM and EWM fused images with SE-LRMF have the lowest noise level. Thus, CLC with SE-LRMF is more likely to avoid the bias due to noise. In other words, compared to RPCA and L1-LRMF, SE-LRMF has the potential to achieve a more robust performance in the proposed framework for signal level fusion.

Moreover, we perform another similar analysis. In this analysis, we measure the noise level in LRM and EWM results using the $\ell_{1}$ distance between the raw feature values (feature values before quantization) extracted from these two results. The smaller the $\ell_{1}$ distance, the lower the noise level. The reason is similar to the one stated in the above analysis via bit inconsistency. In Fig. 6, we plot the distribution of $\ell_{1}$ distances calculated from all the image sets for fusion on the three datasets, with RPCA, L1-LRMF and SE-LRMF for LRA.

It can be seen that the result is similar to the analysis based on bit inconsistency. The distribution is consistent with the noise level in each dataset: for the dataset with a lower noise level, there are more raw feature values with a smaller $\ell_{1}$ distance between LRM and EWM results. Also, among the three LRA algorithms, RPCA has the smallest number of small $\ell_{1}$ distances on all the datasets. It means the fusion result of RPCA has heavier noise, and this explains the observation four above. Finally, compared to RPCA and L1-LRMF, the proposed SE-LRMF leads to more small $\ell_{l}$ distances and fewer large $\ell_{1}$ distances on the three datasets. This means that, compared to RPCA and L1-LRMF, SE-LRMF leads to less noise in fusion results, so it is more likely to avoid the bias due to noise, achieving a more robust performance.

\section{Comparison With No Fusion Methods}

We use iris recognition results without fusion as the baseline. We make the comparisons between the proposed SE-LRMF based signal level fusion (referred as SE-LRMF) and no fusion baseline methods. We design two no fusion methods. No fusion method 1 (NF1) is a single gallery single probe method. It simulates the case that we use one image per eye as the gallery in registration phase, and we capture a single probe image for matching in iris matching stage. The comparison between NF1 and SE-LRMF explores the performance between: (1) only using the information in single capture as gallery and probe and (2) fusing the information in multiple captures to produce gallery and probe. The gallery and probe sets for NF1 are constructed as follows. Recall that in the fusion setting in section IV-A, we have some image sets for fusion to construct gallery and probe sets for information fusion methods. To construct the gallery of NF1, we retrieve all the image sets that are used to construct the gallery in our fusion setting. From each retrieved image set, we randomly select 1 image, and we extract the iris code from the selected image as one NF1 gallery iris code. In other words, given a set of images from the same eye, SE-LRMF fuses them to produce one gallery iris code, while NF1 randomly selects one of them to produce one gallery iris code. It leads to a NF1 gallery set with the same iris code number and label to the SE-LRMF gallery set. We use the same method to produce the probe set of NF1. Given a pair of gallery and probe sets of NF1, we calculate $\mathrm{CMC}$ and ROC curves to evaluate the performance. We repeat the above random selection of gallery and probe sets of NF1 for 50 times. We use the mean of the CMC and ROC curves obtained from the 50 runs as the performance of NF1 for comparison. We note that the above procedure may lead to repeated probe iris codes in the NF1 probe set, since in our 


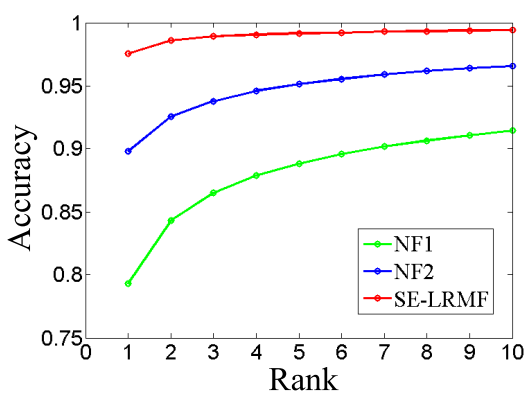

(a)

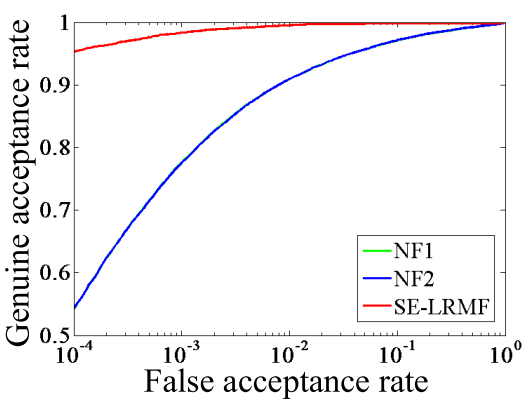

(d)

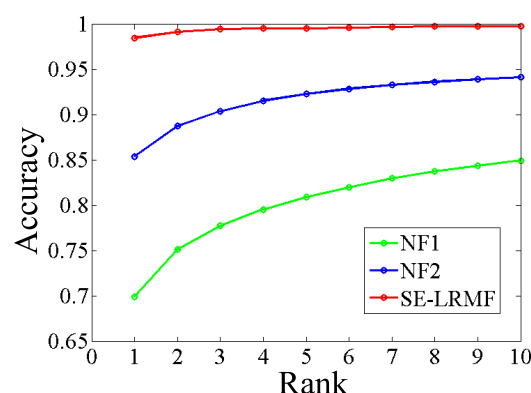

(b)

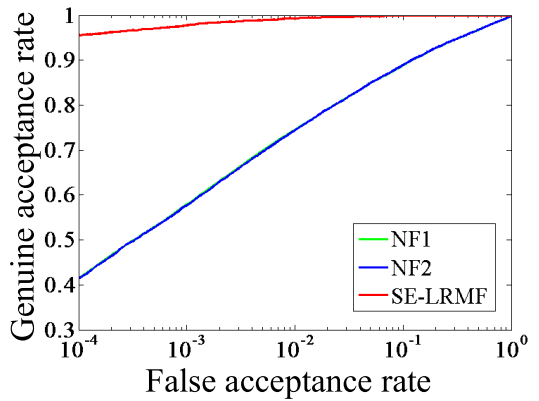

(e)

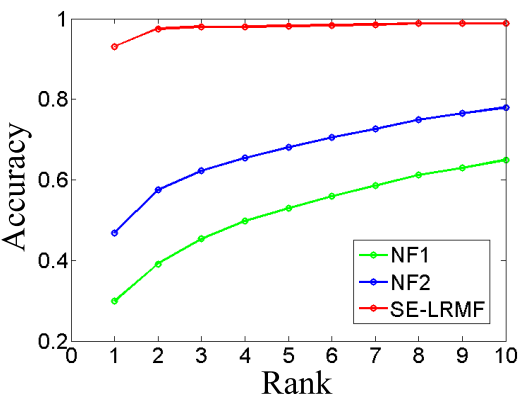

(c)

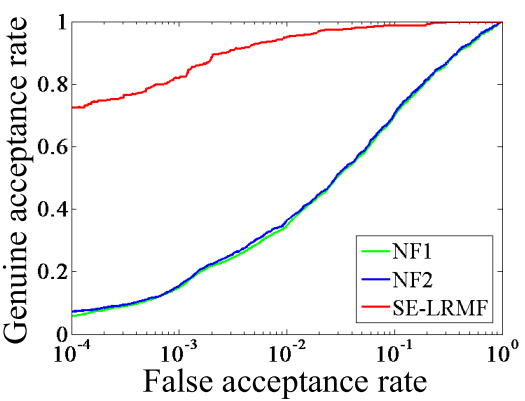

(f)

Fig. 7. The CMC and ROC curves for the comparison between SE-LRMF and no fusion methods. (a) CMC, ND0405. (b) CMC, CASIA4. (c) CMC, UBIRIS2. (d) ROC, ND0405. (e) ROC, CASIA4. (f) ROC, UBIRIS2.

fusion setting, the image sets used to produce the probe set can overlap. However, we think the random selection and multiple runs make NF1 a reasonable estimator of the performance of a single probe single gallery no fusion setting.

No fusion method 2 (NF2) is a multiple gallery single probe method without fusion. It simulates the case that we enrich the information in gallery by capturing multiple iris images per eye but without fusing them. In matching phase, a single probe is captured and compared with the gallery. The probe set of NF2 is constructed using exactly the same method as NF1. As for the gallery set, we retrieve all the image sets used to produce the gallery in our fusion setting, and we use the iris codes extracted from all the retrieved images as gallery. Similar to NF1, the above procedure is repeated 50 times, and we use mean CMC and ROC curves as the estimation of the performance of NF2 for comparison. Note that for NF2, the gallery set is exactly the same in each run, and only the probe set is different due to random selection.

We report in Fig. 7 the CMC and ROC curves of NF1, NF2 and SE-LRMF on all the datasets. It can be seen that: (1) NF2 has a better performance compared to NF1; (2) SE-LRMF leads to a significantly improved performance compared to the two no fusion methods. The results demonstrate that: (1) an improved performance can be achieved by using more gallery images; (2) signal level fusion of the information in multiple captures performs better than using the information in single captures without fusion.

\section{E. Comparison With Existing Signal Level Fusion Methods}

In this subsection, we compare the proposed SE-LRMF based signal level fusion method with existing signal level fusion methods. We make the comparison with mean fusion (MF) proposed in [19] and quality weighted mean (QWM) fusion proposed in [21]. We report CMC and ROC curves of MF, QWM and SE-LRMF in Fig. 8. It can be seen that the proposed SE-LRMF based signal level fusion outperforms existing signal level fusion methods.

\section{F. Comparison With Existing Score Level Fusion Methods}

In this subsection, we make comparisons between the proposed SE-LRMF based signal level fusion method and existing score level fusion methods. We compare with three score level fusion methods: fusion using the mean of scores (score-mean) [19], [24], fusion using the minimum of scores (score-min) [19], [24], fusion using quality weighted mean of scores (score-QWM) [21], [25]. We report the CMC and ROC curves of score-mean, score-min, score-QWM and SE-LRMF in Fig. 9.

Considering the identification performance (CMC) shown in Fig. 9, we find that the performance of different algorithms differs according to the datasets. On ND-iris-0405, mean score based fusion methods (score-mean, score-QWM) achieve higher rank 1 recognition accuracies than SE-LRMF, while SE-LRMF performs better than score-min. On CASIA.v4 distance, the performance of SE-LRMF is comparable to score-min, and it is better than mean score based methods. On UBIRIS.v2, SE-LRMF outperforms all the other methods.

However, despite of the varying performance, we can see that the performance of SE-LRMF is close to the top score level method on less noisy NIR datasets, and it achieves a significantly improved performance on more noisy colour dataset. The rank 1 recognition accuracy of SE-LRMF 


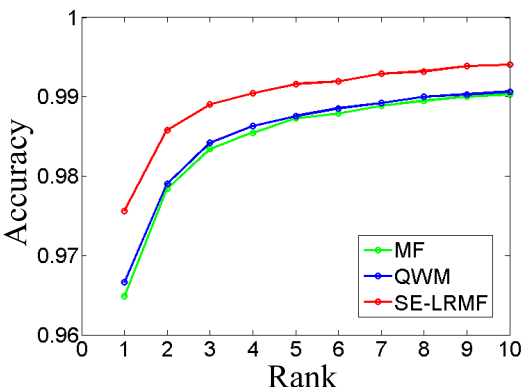

(a)

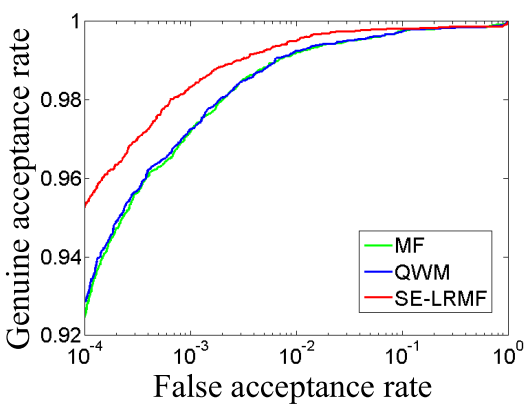

(d)

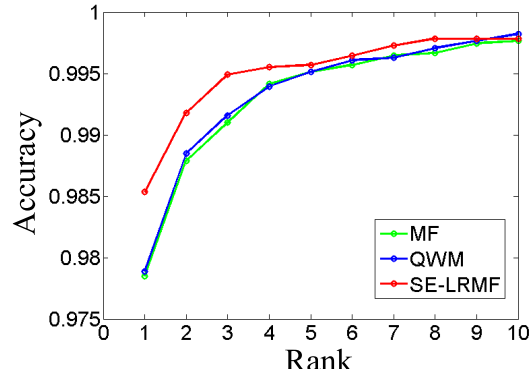

(b)

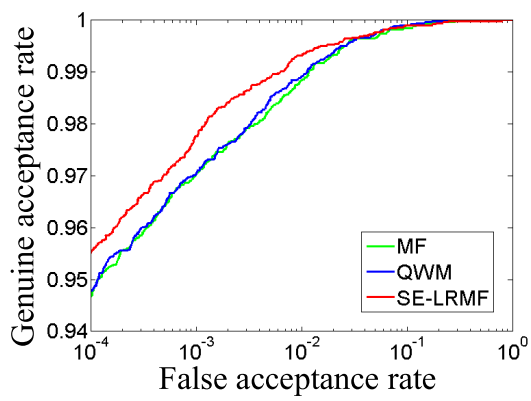

(e)

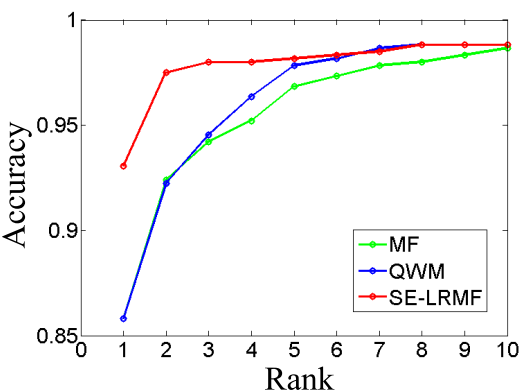

(c)

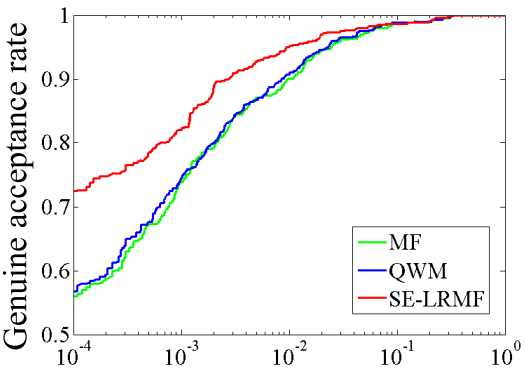

False acceptance rate

(f)

Fig. 8. The CMC and ROC curves for the comparison between SE-LRMF and existing signal level fusion methods. (a) CMC, ND0405. (b) CMC, CASIA4 (c) CMC, UBIRIS2. (d) ROC, ND0405. (e) ROC, CASIA4. (f) ROC, UBIRIS2.

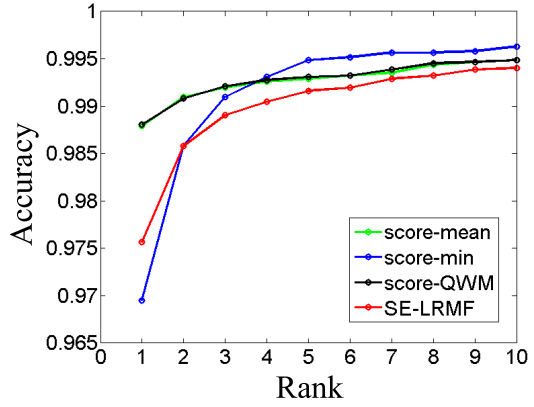

(a)

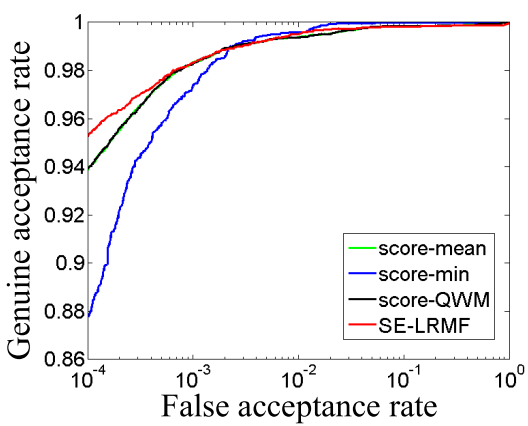

(d)

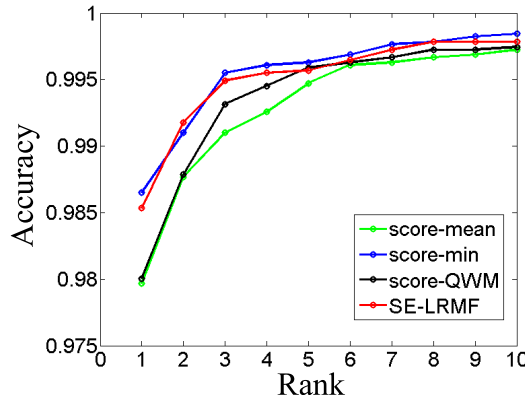

(b)

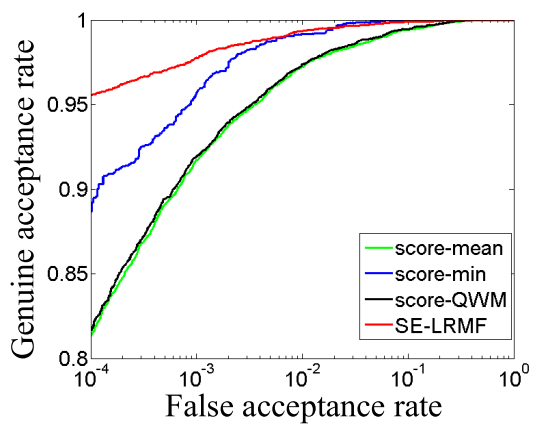

(e)

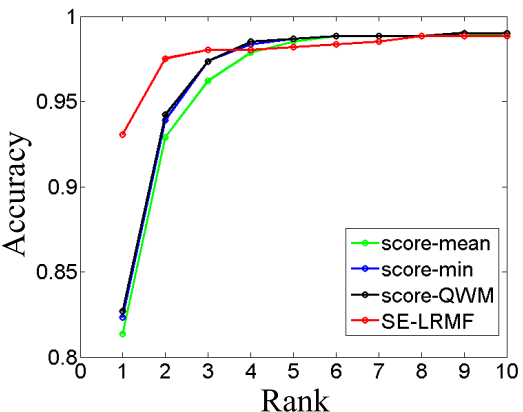

(c)

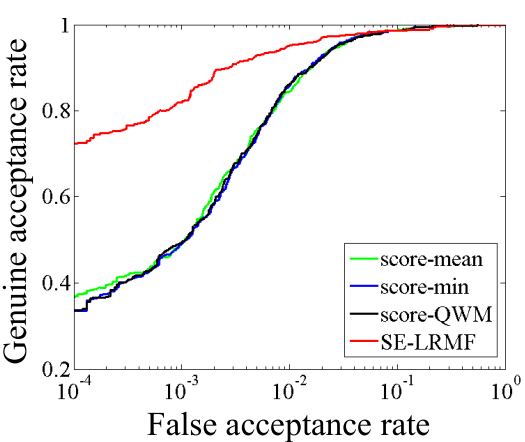

(f)

Fig. 9. The CMC and ROC curves for the comparison between SE-LRMF and existing score level fusion methods. (a) CMC, ND0405. (b) CMC, CASIA4. (c) CMC, UBIRIS2. (d) ROC, ND0405. (e) ROC, CASIA4. (f) ROC, UBIRIS2.

is $1.25 \%$ lower than the best method on ND-iris-0405, and it is $0.12 \%$ to the best method on CASIA.v4 distance. On UBIRIS.v2, the rank 1 recognition accuracy of SE-LRMF is $10.40 \%$ higher than the second best method.

Also, considering the stability of identification performance, we find that SE-LRMF is more stable compared to each individual score level fusion method. We can see from the $\mathrm{CMC}$ cruves that the performance of score level fusion methods is less stable. score-mean and score-QWM perform better than score-min on ND-iris-0405, but score-min performs better on the other two datasets. In contrast, SE-LRMF achieves midlevel performance on the NIR datasets, and it performs best on the colour dataset. 
We think the above identification performance is closelyrelated to the noise level in the iris images in each dataset. Score level fusion is sensitive to the noise level in iris captures. Lower noise level leads to higher reliability in the matching scores between individual gallery and probe iris codes, hence it results more reliable fused scores. On the contrary, if the noise level is too high, the matching scores between individual gallery and probe iris codes become highly unreliable. As a result, fusing these matching scores brings little advantage. Compared to score level fusion, signal level fusion is less sensitive to the noise level of iris captures. The reason is that the iris codes of signal level fusion are extracted from the fused images, and the fusion process is able to suppress noise and enhance the stable structure in iris images.

The above analysis is consistent to the CMC curves in Fig. 9. ND-iris-0405 dataset has a relatively lower noise level. Therefore, on this dataset, the best score level fusion method (score-QWM) performs better than the signal level SE-LRMF. The noise level of the images in CASIA.v4 distance dataset is higher than that in ND-iris-0405 dataset. Correspondingly, on this dataset, signal level SE-LRMF performs similar to the best score level fusion method (score-min). The images in UBIRIS, v2 dataset contain relatively heavier noise due to a combination of visible wavelength and distant capturing. Consequently, signal level SE-LRMF still performs well, while the performance of score level fusion methods heavily degrades.

In terms of verification performance, SE-LRMF achieves better ROC curves compared to score level fusion methods on all the datasets. It is interesting that score level fusion performs good in CMC curves but not in ROC curves. We think such observation can be explained as follows. Score level fusion is more accurate in intra-class matching. It leads to more accurate lowest matching scores and good CMC curves. However, score level fusion is less effective in inter-class comparisons. It results in less accurate inter-class matching scores which influence ROC curves. In contrast, SE-LRMF performs well in both intra-class and inter-class matching, and it leads to good performance in both CMC and ROC curves.

In conclusion, compared to score level fusion, (1) SE-LRMF based signal level fusion is able to achieve a better identification performance on the colour data with relatively heavier noise, while it also performs well on the NIR captures with relatively lower noise; (2) SE-LRMF based signal level fusion is more stable in identification; (3) SE-LRMF based signal level fusion achieves better verification performance on the iris data with varying noise levels.

\section{G. Comparison With Other Recent Iris Recognition Algorithms}

In this subsection, we make comparisons with other recently proposed iris recognition algorithms. These algorithms generally focus on improving the feature extraction and iris matching stages, instead of performing information fusion using multiple captures. This comparison studies the effectiveness of information fusion in less constrained iris recognition.

We compare with two recent algorithms: (1) geometric key-based iris encoding [40] (GeoKey) and (2) stabilized iris encoding and Zernike moments phase features [16] (SZM).
We choose these two algorithms for comparison, since they are recently proposed algorithms achieving top performance compared to other state-of-the-art algorithms in less constrained iris recognition, as reported in [16] and [40]. We also compare with the result of the fusion of the two algorithms by combining their matching scores (referred as GeoKey+SZM).

Considering that the above algorithms are not information fusion algorithms, to make a reasonable comparison, we evaluate their performance using the same experimental setting to the no fusion method 2 (NF2) in section IV-D. Also, we use NF2 as a reference of baseline performance.

We show the CMC and ROC curves for all the comparison algorithms on all the datasets in Fig. 10. It can be seen that GeoKey, SZM and GeoKey+SZM perform significantly better than NF2, while SE-LRMF achieves top performance among all the comparison methods. Actually, nearly all the information fusion algorithms used in our comparisons in previous subsections achieve better performance than GeoKey, SZM and GeoKey+SZM. This result shows that information fusion is able to significantly improve the performance of less constrained iris recognition, even with less robust features (note that the results of SE-LRMF and the information fusion methods used in our comparisons in previous subsections are based on the same feature as NL2). We think the reason is that the robustness of the algorithm is significantly enhanced in the fusion process of signals (scores) from multiple captures.

\section{$H$. The Influence of Noise on the Performance of SE-LRMF}

As shown in the comparison in section IV-F, the performance of SE-LRMF based fusion depends on the noise level of captures. In this subsection, we conduct experiments to gain a deeper insight into the influence of different noise types and noise levels on the performance of SE-LRMF.

In this experiment, we group the images sets for fusion in our fusion setting into several groups based on the noise type and noise level, and we investigate the performance of the fusion result corresponding to each group of image sets. We use some quality measures (see below) to compute the level of different types of noise in the data (e.g. blur, off-angle, etc.). Given an image set for fusion and given a quality measure, we compute the quality of this image set as the mean quality of each image in it. Given the image sets in our fusion setting evaluated by a specific quality measure, we group them into 5 quality levels. The first level includes the image sets with top $20 \%$ best quality; the second level includes the image sets with $21 \%$ to $40 \%$ quality; similarly for the other levels. Therefore, the group with a larger index for quality level has a higher noise level. In this paper, we only perform grouping for the image sets used to produce the probe set in our fusion setting. To evaluate the performance of each group of image sets, we match the probes produced by each group to the full gallery set produced by our fusion setting. It simulates the scenario that, in real applications, the gallery set usually remains unchanged after registration phase, and the variation of noise types and noise levels mainly exist in the probes. 


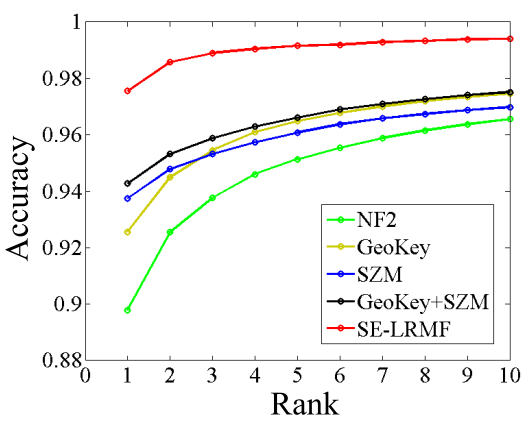

(a)

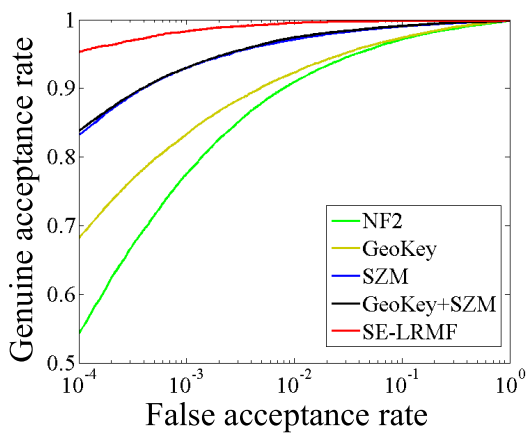

(d)

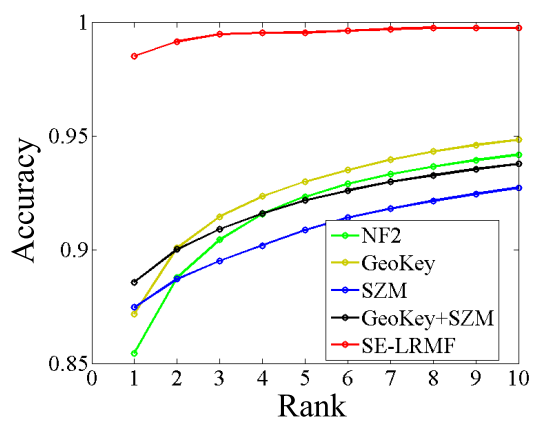

(b)

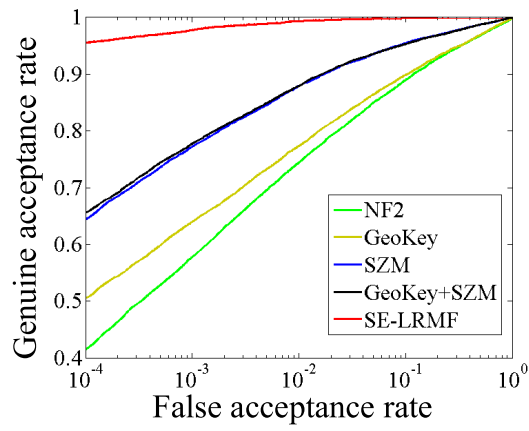

(e)

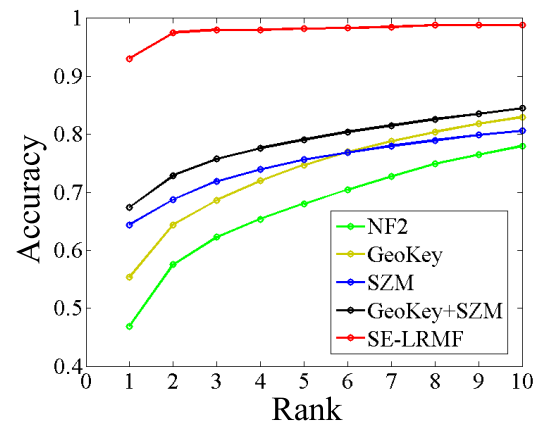

(c)

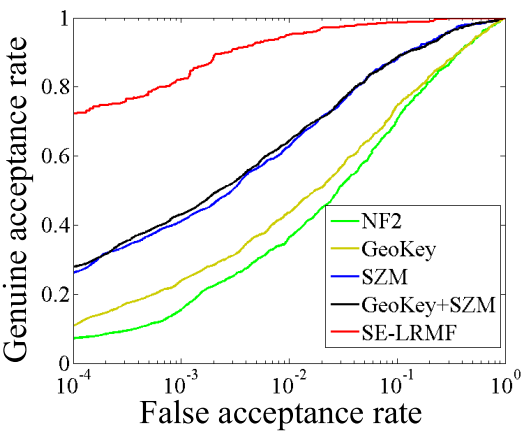

(f)

Fig. 10. The CMC and ROC curves for the comparison between SE-LRMF and other recent iris recognition algorithms. (a) CMC, ND0405. (b) CMC, CASIA4. (c) CMC, UBIRIS2. (d) ROC, ND0405. (e) ROC, CASIA4. (f) ROC, UBIRIS2.

In this paper, we investigate four representative noise types: blur, off-angle, occlusion and motion. We calculate the corresponding quality measures using the method in [21] and [43]. We focus on the comparison between the identification performance of SE-LRMF and score level fusion methods. For the other cases (comparison with signal level fusion and other recent iris recognition algorithms in identification and verification; comparison with score level fusion in verification), we find that SE-LRMF performs generally better than the compared methods across all the levels of different noise types, on the three datasets. It is consistent with the results in previous subsections where only score level fusion performs better than SE-LRMF on some datasets in identification.

We use rank 1 accuracy to represent the identification performance. In Fig. 11, we show the rank 1 accuracy of all the comparison methods on different levels of each quality measure. It can be seen that, on ND0405 which has the lowest noise level, SE-LRMF performs generally worse than the best score level fusion method on all the levels of each quality measure; on CASIA4 with a higher noise level, SE-LRMF performs worse than the best score level fusion method on the first few quality levels, but it performs generally better on the last few quality levels; on UBIRIS2 with the highest noise level, SE-LRMF performs generally better than the other methods across all the quality levels. The result shows that SE-LRMF performs better for the data with a higher noise level.

However, we find that, in Fig. 11, the trend of performance change is not fully consistent with the noise level. In some cases, the performance has obvious perturbation or increasing when the noise level increases (for example, the focus measure on ND0405 and UBIRIS2). In our opinion, this is due to the mixture of noise types. In the datasets we use, a capture usually suffers from multiple types of noise. Consequently, the final performance is influenced by the combination of all these noise types, rather than a specific one. Therefore, given that the image sets are grouped based on one quality measure (i.e. the level of one noise type), the final performance is possible to be influenced by the variation in the level of other noise types which may also affect each group. Also, more complexity exists since noise types may not be independent with each other. We think this problem of mixture of noise types can be addressed in future research, either by performing data capturing with the target noise type varied and the others restricted at low levels, or by combining multiple individual quality measures to obtain a quality measure that is better related to the final performance.

Furthermore, we find that, the performance change is more consistent with the change of motion level. This observation means that the influence of motion on the final performance is more dominant than the other noise types. We think a possible reason is that motion is also source of other noise types, like blur and off-angle. Therefore, the relevant quality measure summarizes more relevant noise types.

In all, despite of the problem of mixture of noise types, SE-LRMF performs better than the other methods on the data with a higher level in any of the four noise types.

\section{CONClusion}

In this paper, we address the problem of iris recognition under less constrained environments. We propose a signal level fusion method based on low rank approximation. First, we propose a sparse-error low rank matrix factorization (SE-LRMF) model to separate iris pixels and noise pixels in multiple 


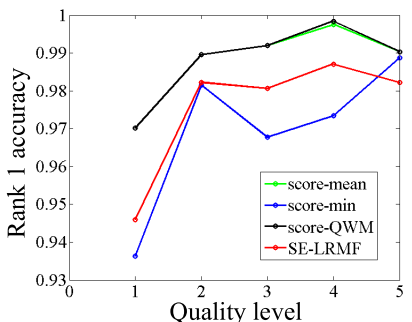

(a)

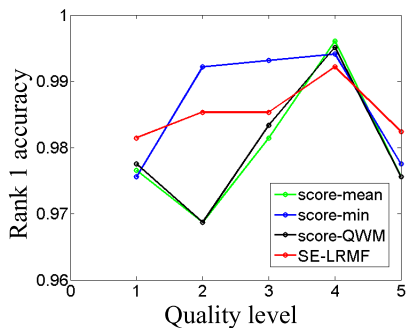

(e)

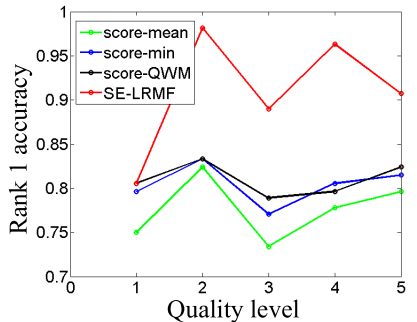

(i)

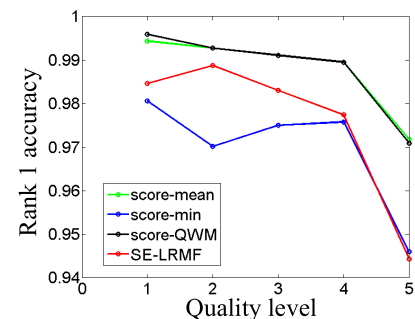

(b)

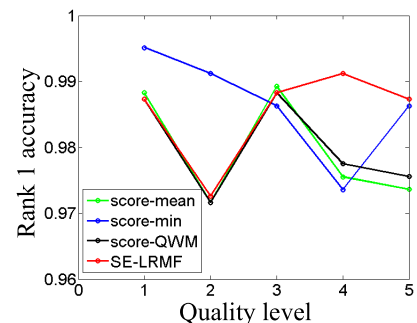

(f)

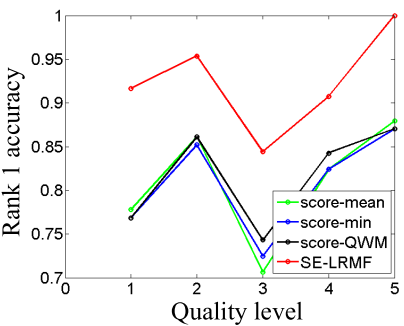

(j)

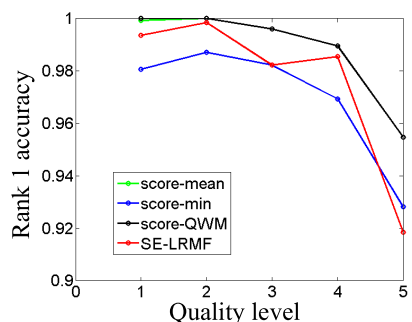

(c)

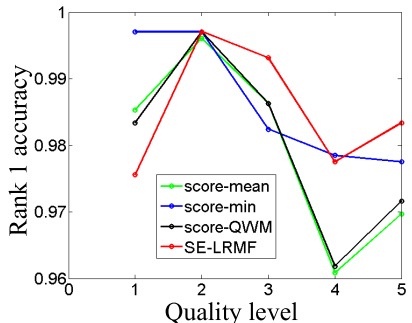

(g)

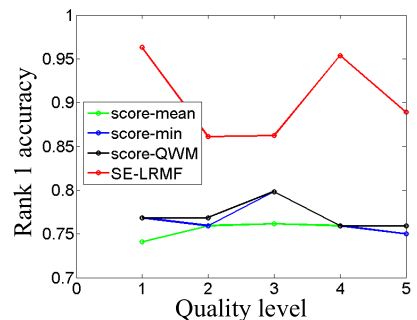

(k)

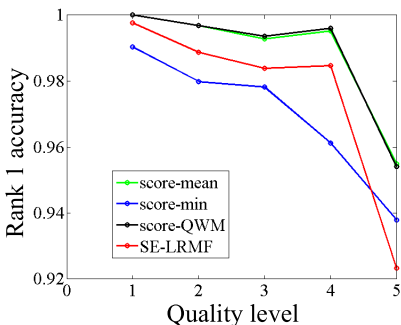

(d)

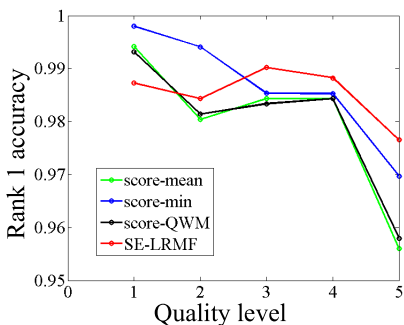

(h)

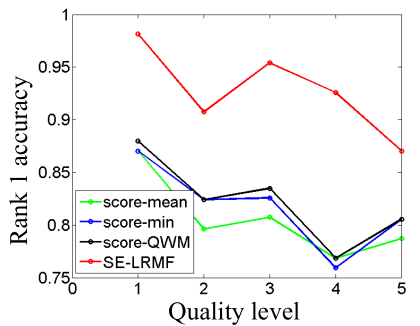

(1)

Fig. 11. The performance of SE-LRMF and score level fusion methods on varying levels of different quality measures. (a) ND0405, focus. (b) ND0405, off-angle. (c) ND0405, occlusion. (d) ND0405, motion. (e) CASIA4, focus. (f) CASIA4, off-angle. (g) CASIA4, occlusion. (h) CASIA4, motion. (i) UBIRIS2, focus. (j) UBIRIS2, off-angle. (k) UBIRIS2, occlusion. (1) UBIRIS2, motion.

iris captures. Then, the iris pixels and noise pixels are utilized to perform signal level fusion individually, leading to a low rank mean (LRM) fusion method and an error weighted mean (EWM) fusion method. Finally, the results of LRM and EWM are combined at the code level to produce a final iris code. We conduct experiments on benchmark iris datasets captured under different wavelengths and distances, with varying noise types and noise levels. The main conclusions are summarized as follows:

- Using SE-LRMF for low rank approximation, the code level combination of LRM and EWM results leads to an improved performance over individual LRM and EWM results.

- In comparison to representative low rank approximation algorithms, SE-LRMF leads to a better and more stable performance in the proposed framework of signal level fusion. Also, SE-LRMF has the potential to achieve a more robust performance.

- The proposed SE-LRMF based signal level fusion leads to a significantly improved performance compared to the baseline algorithms without fusion. It also performs better than recently proposed iris recognition algorithms that do not fuse the information from multiple captures.

- The proposed SE-LRMF based signal level fusion outperforms existing signal level fusion methods.

- Compared to existing score level fusion methods, the proposed SE-LRMF based signal level fusion has a competitive and more stable identification performance, and a better verification performance.

- The proposed SE-LRMF based signal level fusion performs better for the captures with relatively heavier noise.

Future work may consider to incorporate quality measures into the proposed method to achieve better performance.

\section{REFERENCES}

[1] J. G. Daugman, "High confidence visual recognition of persons by a test of statistical independence," IEEE Trans. Pattern Anal. Mach. Intell., vol. 15, no. 11, pp. 1148-1161, Nov. 1993.

[2] J. Daugman, "How iris recognition works," IEEE Trans. Circuits Syst. Video Technol., vol. 14, no. 1, pp. 21-30, Jan. 2004.

[3] R. P. Wildes, "Iris recognition: An emerging biometric technology," Proc. IEEE, vol. 85, no. 9, pp. 1348-1363, Sep. 1997.

[4] K. W. Bowyer, K. Hollingsworth, and P. J. Flynn, "Image understanding for iris biometrics: A survey," Comput. Vis. Image Understand., vol. 110, no. 2, pp. 281-307, 2008.

[5] Z. He, T. Tan, Z. Sun, and X. Qiu, "Toward accurate and fast iris segmentation for iris biometrics," IEEE Trans. Pattern Anal. Mach. Intell., vol. 31, no. 9, pp. 1670-1684, Sep. 2009.

[6] A. Kumar and A. Passi, "Comparison and combination of iris matchers for reliable personal authentication," Pattern Recognit., vol. 43, no. 3, pp. 1016-1026, 2010.

[7] W. Dong, Z. Sun, and T. Tan, "Iris matching based on personalized weight map," IEEE Trans. Pattern Anal. Mach. Intell., vol. 33, no. 9, pp. 1744-1757, Sep. 2011.

[8] K. P. Hollingsworth, K. W. Bowyer, and P. J. Flynn, "The best bits in an iris code," IEEE Trans. Pattern Anal. Mach. Intell., vol. 31, no. 6, pp. 964-973, Jun. 2009.

[9] P. Li and G. Wu, "Iris recognition using ordinal encoding of LogEuclidean covariance matrices," in Proc. IEEE 21st Int. Conf. Pattern Recognit., vol. 31. Nov. 2012, pp. 2420-2423. 
[10] J. K. Pillai, V. M. Patel, R. Chellappa, and N. K. Ratha, "Secure and robust iris recognition using random projections and sparse representations," IEEE Trans. Pattern Anal. Mach. Intell., vol. 33, no. 9, pp. 1877-1893, Sep. 2011.

[11] H. Proença and L. A. Alexandre, "Toward covert iris biometric recognition: Experimental results from the NICE contests," IEEE Trans. Inf. Forensics Security, vol. 7, no. 2, pp. 798-808, Apr. 2012.

[12] H. Proença, S. Filipe, R. Santos, J. Oliveira, and L. A. Alexandre, "The UBIRIS.v2: A database of visible wavelength iris images captured onthe-move and at-a-distance," IEEE Trans. Pattern Anal. Mach. Intell., vol. 32, no. 8, pp. 1529-1535, Jun. 2010.

[13] Z. Sun and T. Tan, "Ordinal measures for iris recognition," IEEE Trans. Pattern Anal. Mach. Intell., vol. 31, no. 12, pp. 2211-2226, Dec. 2009.

[14] C.-W. Tan and A. Kumar, "Unified framework for automated iris segmentation using distantly acquired face images," IEEE Trans. Image Process., vol. 21, no. 9, pp. 4068-4079, Sep. 2012.

[15] C.-W. Tan and A. Kumar, "Adaptive and localized iris weight map for accurate iris recognition under less constrained environments," in Proc. IEEE Int. Conf. Biometrics Theory, Appli., Syst., Sep./Oct. 2013, pp. 1-7.

[16] C.-W. Tan and A. Kumar, "Accurate iris recognition at a distance using stabilized iris encoding and Zernike moments phase features," IEEE Trans. Image Process., vol. 23, no. 9, pp. 3962-3974, Sep. 2014.

[17] J. R. Matey et al., "Iris on the move: Acquisition of images for iris recognition in less constrained environments," Proc. IEEE, vol. 94, no. 11, pp. 1936-1947, Nov. 2006.

[18] S. Venugopalan et al., "Long range iris acquisition system for stationary and mobile subjects," in Proc. IEEE Int. Joint Conf. Biometrics, Oct. 2011, pp. 1-8.

[19] K. Hollingsworth, T. Peters, K. W. Bowyer, and P. Flynn, "Iris recognition using signal-level fusion of frames from video," IEEE Trans. Inf. Forensics Security, vol. 4, no. 4, pp. 837-848, Dec. 2009.

[20] K. Nguyen, C. Fookes, S. Sridharan, and S. Denman, "Focus-score weighted super-resolution for uncooperative iris recognition at a distance and on the move," in Proc. 25th Int. Conf. Image Vis. Comput., 2010, pp. $1-8$.

[21] K. Nguyen, C. Fookes, S. Sridharan, and S. Denman, "Quality-driven super-resolution for less constrained iris recognition at a distance and on the move," IEEE Trans. Inf. Forensics Security, vol. 6, no. 4, pp. 1248-1258, Dec. 2011 .

[22] K. Nguyen, C. Fookes, S. Sridharan, and S. Denman, "Feature-domain super-resolution for iris recognition," in Proc. 18th IEEE Int. Conf. Image Process., Sep. 2011, pp. 3197-3200.

[23] K. Nguyen, S. Sridharan, S. Denman, and C. Fookes, "Feature-domain super-resolution framework for Gabor-based face and iris recognition," in Proc. IEEE Conf. Comput. Vis. Pattern Recognit., Jun. 2012, pp. 2642-2649.

[24] J. Liu, Z. Sun, and T. Tan, "Code-level information fusion of lowresolution iris image sequences for personal identification at a distance," in Proc. IEEE 6th Int. Conf. Biometrics Theory, Appl. Syst., Sep./Oct. 2013, pp. 1-6.

[25] K. Nguyen, C. Fookes, S. Sridharan, and S. Denman, "Feature-domain super-resolution for iris recognition," Comput. Vis. Image. Understand., vol. 117, no. 10, pp. 1526-1535, 2013.

[26] E. Candes, X. Li, Y. Ma, and J. Wright, "Robust principal component analysis?" J. ACM, vol. 58, no. 3, 2011, Art. no. 13.

[27] D. Meng, Z. Xu, L. Zhang, and J. Zhao, "A cyclic weighted median method for $L_{1}$ low-rank matrix factorization with missing entries," in Proc. 27th AAAI Conf. Artif. Intell., 2013, pp. 704-710.

[28] T. Okatani and K. Deguchi, "On the Wiberg algorithm for matrix factorization in the presence of missing components," Int. J. Comput. Vis., vol. 72, no. 3, pp. 329-337, Mar. 2007.

[29] N. Srebro and T. Jaakkola, "Weighted low-rank approximations," in Proc. Int. Conf. Mach. Learn., 2003, pp. 720-727.

[30] K. Y. Shin, K. R. Park, B. J. Kang, and S. J. Park, "Super-resolution method based on multiple multi-layer perceptrons for iris recognition," in Proc. 4th Int. Conf. Ubiquitous Inf. Technol. Appl., Dec. 2009, pp. 1-5.

[31] A. M. Buchanan and A. W. Fitzgibbon, "Damped Newton algorithms for matrix factorization with missing data," in Proc. IEEE Comput. Soc. Conf. Comput. Vis. Pattern Recognit., vol. 2. Jun. 2005, pp. 316-322.

[32] T. Wiberg, "Computation of principal components when data are missing," in Proc. 2nd Symp. Comput. Statist., 1976, pp. 229-236.

[33] A. Eriksson and A. van den Hengel, "Efficient computation of robust low-rank matrix approximations in the presence of missing data using the $L_{1}$ norm," in Proc. IEEE Conf. Comput. Vis. Pattern Recognit., Jun. 2010, pp. 771-778.
[34] C. Ding, D. Zhou, X. He, and H. Zha, "R1-PCA: Rotational invariant $L_{1}$-norm principal component analysis for robust subspace factorization," in Proc. 23rd Int. Conf. Mach. Learn., 2006, pp. 281-288.

[35] N. Kwak, "Principal component analysis based on $L_{1}$-norm maximization," IEEE Trans. Pattern Anal. Mach. Intell., vol. 30, no. 9, pp. $1672-1680$, Sep. 2008 .

[36] E. T. Hale, W. Yin, and Y. Zhang, "Fixed-point continuation for $L_{1}$-minimization: Methodology and convergence," SIAM J. Optim., vol. 19 , no. 3, pp. 1107-1130, 2008.

[37] P. J. Phillips et al., "FRVT 2006 and ICE 2006 large-scale experimental results," IEEE Trans. Pattern Anal. Mach. Intell., vol. 32, pp. 831-846, 2010.

[38] Biometrics Ideal Test, accessed on Oct. 14, 2014. [Online]. Available: http://biometrics.idealtest.org/dbDetailForUser.do?id=4

[39] P. Viola and M. Jones, "Rapid object detection using a boosted cascade of simple features," in Proc. IEEE Conf. Comput. Vis. Pattern Recognit., Dec. 2001, pp. I-511-I-518.

[40] C. W. Tan and A. Kumar, "Efficient and accurate at-a-distance iris recognition using geometric key-based iris encoding," IEEE Trans. Inf. Forensics Security, vol. 9, no. 9, pp. 1518-1526, Sep. 2014.

[41] Y. Hu, K. Sirlantzis, and G. Howells, "A robust algorithm for colour iris segmentation based on 1-norm regression," in Proc. IEEE Int. Joint Conf. Biometrics, Sep./Oct. 2014, pp. 1-8.

[42] L. Masek and P. Kovesi. (2003). MATLAB source code for a biometric identification system based on iris patterns. School Comput. Sci. Softw. Eng., Univ. Western Australia, Perth, WA, Australia. [Online]. Available: http://staffhome.ecm.uwa.edu.au/ 00011811/studentprojects/libor/ sourcecode.html

[43] H. Proença, "Quality assessment of degraded iris images acquired in the visible wavelength," IEEE Trans. Inf. Forensics Security, vol. 6, no. 1, pp. 82-95, Mar. 2011.

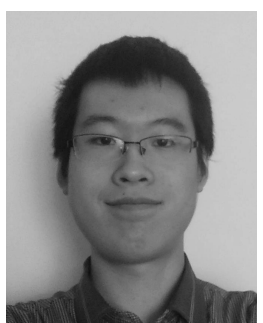

Yang $\mathrm{Hu}$ received the B.Sc. degree in electronics and information engineering from Dalian Maritime University, China, in 2010, and the M.Sc. degree in engineering from the Dalian University of Technology, China, in 2013. He is currently pursuing the Ph.D. degree in electronics engineering with the University of Kent, U.K. His research interest focuses on biometrics, computer vision, and pattern recognition.

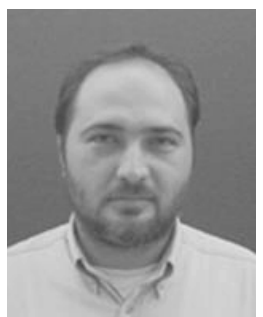

Konstantinos Sirlantzis is currently a Lecturer in Image Processing and Vision with the School of Engineering and Digital Arts, University of Kent. He has a strong track record in image analysis and understanding, artificial intelligence, and neural networks for pattern recognition and biometrics-based security applications. He leads a research team working on social assistive and autonomous robotics and health-related assistive technologies. He organized and chaired a range of international conferences and workshops, such as RASC 2006 and Emerging Security Technologies 2010. He has authored over 100 peer-reviewed papers in journals and conferences.

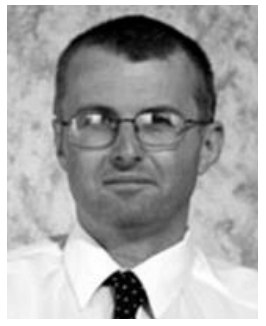

Gareth Howells is a Reader in Secure Electronic Systems with the University of Kent, U.K. He has been involved in research relating to security, biometrics, and pattern classification techniques for over 25 years and has been instrumental in the development of novel ICMetric-based security technology deriving secure encryption keys from the operating characteristics of digital systems. He has been awarded, either individually or jointly, several major research grants relating to the pattern classification and security fields, publishing over 180 papers in the technical literature. His recent work has been directed toward the development of secure device authentication systems which has received significant funding from several funding bodies and is currently in the process of being commercially exploited. 